\title{
RANS-based simulation of wave-induced sheet-flow transport of graded sediments
}

\author{
Calistan, Ugur; Fuhrman, David R.
}

Published in:

Coastal Engineering

Link to article, DOI:

10.1016/j.coastaleng.2016.11.007

Publication date:

2017

Document Version

Peer reviewed version

Link back to DTU Orbit

Citation (APA):

Calistan, U., \& Fuhrman, D. R. (2017). RANS-based simulation of wave-induced sheet-flow transport of graded sediments. Coastal Engineering, 121, 90-102. https://doi.org/10.1016/j.coastaleng.2016.11.007

\section{General rights}

Copyright and moral rights for the publications made accessible in the public portal are retained by the authors and/or other copyright owners and it is a condition of accessing publications that users recognise and abide by the legal requirements associated with these rights.

- Users may download and print one copy of any publication from the public portal for the purpose of private study or research.

- You may not further distribute the material or use it for any profit-making activity or commercial gain

- You may freely distribute the URL identifying the publication in the public portal

If you believe that this document breaches copyright please contact us providing details, and we will remove access to the work immediately and investigate your claim. 


\title{
RANS-based simulation of wave-induced sheet-flow transport of graded sediments
}

\author{
Ugur Caliskan, David R. Fuhrman* \\ Technical University of Denmark, Department of Mechanical Engineering, DK-2800 Kgs. Lyngby, Denmark
}

\begin{abstract}
A one-dimensional vertical (1DV) turbulence-closure flow model, coupled with sediment transport capabilities, is extended to incorporate graded sediment mixtures. The hydrodynamic model solves the horizontal component of the incompressible Reynolds-averaged Navier-Stokes (RANS) equations coupled with $k-\omega$ turbulence closure. The sediment transport description includes both bed and suspended load descriptions. So-called high-concentration effects (turbulence damping and hindered settling velocities) are likewise included. The sediment transport model treats the bed and suspended load individually for each grain fraction, including effects associated with increased exposure of larger particles within a mixture. The suspended sediment transport model also makes use of modified reference concentration approach, wherein reference concentrations computed individually for each fraction are translated to a common level, conveniently enabling use of a single computational grid for the simulation of suspended sediments. Parametric study shows that these two effects combine to help alleviate an otherwise systematic tendency towards over- (under) predicted transport rates for fine (coarse) sand fractions. The sediment transport model is validated against sheet-flow experimental oscillatory tunnel measurements beneath velocityskewed wave signals, and demonstrates similar accuracy (transport rates generally within a factor of two) for both graded and uniform sands. The model is likewise validated against an extensive data set involving sheet-flow transport beneath acceleration-skewed wave signals (limited to uniform sands); It is then utilized to study potential effects of gradation on the net transport beneath such flows. The simulations suggest that gradation effects can both increase, as well as decrease, the total transport rate, depending largely on the behavior of the fine sand fraction. The model is implemented within the Matlab environment, and is freely available upon request to the corresponding author.
\end{abstract}

Keywords: Sediment transport, graded sediments, non-uniform sediment mixtures, wave boundary layer, $k-\omega$ model

\section{Introduction}

In nearshore coastal environments under storm conditions bed ripples are typically washed out, and sediments are typically transported within a thin $\mathrm{O}(1 \mathrm{~cm})$ layer above

\footnotetext{
${ }^{*}$ Corresponding author

Email address: drf@mek.dtu.dk (David R. Fuhrman)
} 
the bed (e.g. Hassan and Ribberink, 2010). Cross-shore sediment transport processes under these so-called sheet flow conditions have been the focus of much recent experimental and numerical work. Extensive experimental work in oscillating tunnel facilities have lead to the databases presented by e.g. van der Werf et al. (2009) and Silva et al. (2011). Models for predicting sheet-flow sediment transport rates induced by waves vary widely in their complexity, commonly ranging from: 1) relatively simple quasi-steady approaches (e.g. Nielsen and Callaghan, 2003), 2) intermediately-complex turbulence closure-based methods (e.g. Davies and Li, 1997; Guizien et al., 2003; Holmedal and Myrhaug, 2006, 2009; Ruessink et al., 2009; Hassan and Ribberink, 2010; Blondeaux et al., 2012; Fuhrman et al., 2013), to 3) very detailed two-phase approaches (e.g. Amoudry et al., 2008), which solve momentum equations for water and sand phases separately, including coupling forces. The advantage of turbulence-closure based methodologies over simpler practical approaches is that they provide a more complete description of the coupled flow and sediment transport process (e.g. involving directly calculated bed shear stresses, as well as providing the vertical distribution of turbulence and suspended sediment concentrations), while still maintaining reasonable computational efficiency. As such, when they are coupled directly with advanced process-based hydrodynamic models, they in principal account for the suspension, advection, diffusion, and settling of sediments that occur simultaneously within e.g. cross-shore sediment transport dynamics (e.g. Jacobsen and Fredsøe, 2014) and scour (e.g. Liang and Cheng, 2005; Fuhrman et al., 2014; Baykal et al., 2015) processes.

Most research involving sediment transport beneath waves has focused on experiments or numerical models based on uniform (well-sorted) sediments, with relatively limited emphasis on related processes involving graded sediments. Experimental work involving transport of graded sands beneath velocity-skewed wave signals in oscillatory tunnel environments include those of e.g. Inui et al. (1995), Hamm et al. (1998), O'Donoghue and Wright (2004a,b), and Hassan and Ribberink (2005). Similar experimental work involving graded sediment transport beneath asymmetric waves (acceleration-skewed) signals is seemingly lacking in the literature, though this has been studied for well-sorted sands by e.g. Watanabe and Sato (2004) and van der A et al. (2010). Methods for modeling wave-induced transport of graded sediments include the works of van Rijn (2007) who highlighted the importance of incorporating hiding/exposure corrections factors for calculating transport rates on graded sediment beds, as well as the practical approaches developed recently by e.g. van der A et al. (2013) and $\mathrm{Wu}$ and Lin (2014). To date, published attempts at 1DV turbulence closure based modeling of graded sediments beneath waves are rather limited, seemingly to that of Li and Davies (2001), on which the present paper builds.

The present paper is organized as follows: A brief description of the hydrodynamic and turbulence closure models is provided in $\S 2$, whereas description of the sediment transport model is presented in $\S 3$. Comparisons with experiments involving sediment transport beneath velocity-skewed wave signals are presented in $\S 4$. These results are likewise complemented by a systematic parametric study. Comparisons with experiments involving sediment transport beneath asymmetric (acceleration-skewed) wave signals are presented in $§ 5$. These simulations are complemented by additional simulations, specifically investigating potential effects of sediment gradation beneath such flows. Conclusions are finally summarized in $\S 6$. 


\section{Hydrodynamic model description}

\subsection{Governing equations}

The hydrodynamic model solves the horizontal component of the incompressible Reynoldsaveraged Navier-Stokes (RANS) equations

$$
\frac{\partial u}{\partial t}=-\frac{1}{\rho} \frac{\partial p}{\partial x}+\nu \frac{\partial^{2} u}{\partial y^{2}}+\frac{\partial}{\partial y}\left(\nu_{T} \frac{\partial u}{\partial y}\right)
$$

where $u$ and $v$ are the velocity components in the (horizontal) $x$ and (vertical) $y$ directions, respectively, $t$ is time, $p$ is the pressure, $\rho$ the water density, and $\nu$ the kinematic viscosity.

To achieve closure, the RANS equation is coupled with the two-equation $k-\omega$ turbulence model of Wilcox $(2006,2008)$ :

$$
\begin{gathered}
\frac{\partial k}{\partial t}=\nu_{T}\left(\frac{\partial u}{\partial y} \frac{\partial u}{\partial y}\right)-\beta^{*} k \omega+\frac{\partial}{\partial y}\left[\left(\nu+\sigma^{*} \frac{k}{\omega}\right) \frac{\partial k}{\partial y}\right]-B \\
\frac{\partial \omega}{\partial t}=\alpha \frac{\omega}{k} \nu_{T}\left(\frac{\partial u}{\partial y} \frac{\partial u}{\partial y}\right)-\beta \omega^{2}+\frac{\partial}{\partial y}\left[\left(\nu+\sigma \frac{k}{\omega}\right) \frac{\partial \omega}{\partial y}\right] \\
+\frac{\sigma_{d}}{\omega} \frac{\partial k}{\partial y} \frac{\partial \omega}{\partial y}-c_{3 \omega} N^{2} .
\end{gathered}
$$

Here

$$
k=\frac{1}{2}\left(\overline{u^{\prime 2}}+\overline{v^{\prime 2}}+\overline{w^{\prime 2}}\right)
$$

is the turbulent kinetic energy (per unit mass), where the prime superscripted variables are turbulent velocity fluctuations, with the overbar representing ensemble-averaging, and $\omega$ is the specific dissipation rate. In (3)

$$
\sigma_{d}=\mathcal{H}\left\{\frac{\partial k}{\partial y} \frac{\partial \omega}{\partial y}\right\} \sigma_{d o}
$$

where $\mathcal{H}\{\cdot\}$ is the Heaviside step function, taking value of zero when the argument is negative, and a value of unity otherwise. The eddy viscosity is defined by

$$
\nu_{T}=\frac{k}{\tilde{\omega}}, \quad \tilde{\omega}=\max \left\{\omega, C_{l i m} \frac{|\partial u / \partial y|}{\sqrt{\beta^{*}}}\right\},
$$

where $C_{\text {lim }}=7 / 8$. The default model closure coefficients suggested by Wilcox (2006) are utilized: $\alpha=13 / 25, \beta=\beta_{0} f_{\beta}, \beta_{0}=0.0708, \beta^{*}=9 / 100, \sigma=1 / 2, \sigma^{*}=3 / 5, \sigma_{d o}=1 / 8$. For two-dimensional flows, as considered herein, $f_{\beta}=1$.

The final terms in (2) and (3) describe turbulence suppression, due to density gradients in the fluid-sediment mixture. These terms are implemented analogously to recent $k$ - $\epsilon$ modeling undertaken by Ruessink et al. (2009), where the buoyancy flux is

$$
B=\frac{\nu_{T}}{\sigma_{p}} N^{2}
$$


The square of the so-called Brunt-Vaisala frequency $N$ is calculated according to:

$$
N^{2}=-\frac{g}{\rho_{m}} \frac{\partial \rho_{m}}{\partial y}=-g(s-1) \frac{\partial c}{\partial y}+O(c),
$$

where $\rho_{m}=s \rho c+\rho(1-c)$ is the density of the fluid-sediment mixture, $s=2.65$ is the relative density of the sediment, and $c$ the total suspended sediment concentration. In the present model, the leading-order approximation indicated as the final right-hand-side in (8) is utilized, following Hassan and Ribberink (2010) and Fuhrman et al. (2013). The closure coefficients for this term correspond to: $\sigma_{p}=0.7$, with $c_{3 \omega}=1$ for $N^{2}<=0$ and $c_{3 \omega}=0$ for $N^{2}>0$. Inclusion of these turbulence suppression terms, while not standard, can be extremely important for flows giving rise to high suspended sediment concentrations near the bed.

\subsection{Boundary conditions}

The above equations are solved starting from motionless initial conditions, subject to the following boundary conditions. A frictionless rigid lid is imposed at the top boundary, whereby vertical derivatives of $u, k$, and $\omega$ are set to zero. Alternatively, the bottom boundary is considered a friction wall, and a no-slip boundary condition is imposed, i.e. velocity variables $u$ and $v$ are set to zero.

At the bottom wall a zero-gradient condition is imposed for the turbulent kinetic energy density $k$ i.e. $d k / d y=0$, corresponding to a zero flux of turbulent kinetic energy through the sea bed. This condition is supported by experimental measurements for steady flows on rough beds (Sumer et al., 2003; Fuhrman et al., 2010), while also offering numerical advantages on rough beds (Fuhrman et al., 2010).

The bottom boundary condition for $\omega$ is adopted from Wilcox (2006), where

$$
\omega=\frac{U_{f}^{2}}{\nu} S_{R}, \quad y=0 .
$$

The factor $S_{R}$ is based on the roughness Reynolds number $k_{N}^{+}=k_{N} U_{f} / \nu$, where $k_{N}=$ $2.5 d_{50}$ is Nikuradse's equivalent sand grain roughness ( $d_{50}$ being the median sediment grain diameter), and $U_{f}=\sqrt{\left|\tau_{b}\right| / \rho}$ the instantaneous friction velocity, according to

$$
S_{R}= \begin{cases}\left(\frac{200}{k_{N}^{+}}\right)^{2}, & k_{N}^{+} \leq 5 \\ \frac{K_{r}}{k_{N}^{+}}+\left[\left(\frac{200}{k_{N}^{+}}\right)^{2}-\frac{K_{r}}{k_{N}^{+}}\right] e^{5-k_{N}^{+},}, & k_{N}^{+}>5 .\end{cases}
$$

The value $K_{r}=180$ is utilized herein, as suggested by Fuhrman et al. (2010) in conjunction with the $\partial k / \partial y=0$ bottom boundary condition.

\subsection{Pressure gradient}

A prescribed pressure gradient in (1) is used to drive the flow within the model domain. To obtain a desired free stream velocity signal $u_{0}$, this has been implemented generally as

$$
\frac{1}{\rho} \frac{\partial p}{\partial x}=-\frac{\partial u_{0}}{\partial t}
$$


In the present model two wave shape options are included. The first corresponds to a skewed free-stream velocity having the form of a second-order Stokes wave signal:

$$
\begin{gathered}
u_{0}=U_{1 m} \sin \left(\omega t^{\prime}\right)-U_{2 m} \cos \left(2 \omega t^{\prime}\right) \\
\frac{\partial u_{0}}{\partial t}=U_{1 m} \omega \cos \left(\omega t^{\prime}\right)+2 U_{2 m} \omega \sin \left(2 \omega t^{\prime}\right)
\end{gathered}
$$

where $t^{\prime}=t+t_{0}$, with $t_{0}$ representing a time shift in the signal, which is determined automatically to ensure that $u_{0}(t=0)=0$ for consistency with the motionless initial conditions. In (12) and (13), and hereafter, the variable $\omega=2 \pi / T$ will refer to the wave angular frequency, where $T$ is the wave period. The second option corresponds to the more flexible wave form shape proposed by Abreu et al. (2010):

$$
\begin{gathered}
u_{0}=U_{w} \sqrt{1-r^{2}} \cdot \frac{\sin \left(\omega t^{\prime}\right)+\frac{r \sin \phi}{1+\sqrt{1-r^{2}}}}{1-r \cos \left(\omega t^{\prime}+\phi\right)}, \\
\frac{\partial u_{0}}{\partial t}=U_{w} \omega \sqrt{1-r^{2}} . \\
\frac{\cos \left(\omega t^{\prime}\right)-r \cos \phi-\frac{r^{2}}{1+\sqrt{1-r^{2}}} \sin \phi \sin \left(\omega t^{\prime}+\phi\right)}{\left(1-r \cos \left(\omega t^{\prime}+\phi\right)\right)^{2}},
\end{gathered}
$$

This option allows forcing wave-like velocity signals ranging from sinusoidal (with $r=0$ ) to highly skewed (with $\phi=-\pi / 2$ ) or front-back asymmetric (with $\phi=0$ ) (e.g. Fuhrman et al., 2013).

\section{Sediment transport model description}

\subsection{Bed load model}

The present model extends the uniform grain size sediment transport capabilities described in Fuhrman et al. (2013) to handle multiple grain fractions, each of which is treated individually, following an approach reasonably similar to Li and Davies (2001), though differing in detail. In the present approach, the rate of bed load transport for the $i$ th grain fraction, $q_{B, i}$, is calculated based on the formula of Engelund and Fredsøe (1976):

$$
\Phi_{B, i}=\frac{q_{B, i}}{\sqrt{(s-1) g d_{i}^{3}}}=5 p_{i}\left[\sqrt{\theta_{i}}-0.7 \sqrt{\theta_{c}}\right],
$$

where

$$
p_{i}=w_{f, i}\left[1+\left(\frac{\pi \mu_{d}}{6\left(\theta_{i}-\theta_{c}\right)}\right)^{4}\right]^{-\frac{1}{4}}
$$

is the (weighted) probability of moving grains, $\theta_{c}=0.045$ is the critical Shields parameter, and $w_{f, i}$ is the $i$ th weight fraction comprising a graded sediment mixture. Note that, in contrast to the model of Li and Davies (2001), equal fractions are not necessarily assumed. The coefficient of dynamic friction is by default set to $\mu_{d}=0.65$, though the effect of taking 
$\mu_{d}=1$ will also be considered. For each fraction, the effective Shields parameter is defined by

$$
\theta_{i}=\left(\frac{d_{i}}{d_{50}}\right)^{h_{c}}\left(\frac{U_{f}^{2}}{(s-1) g d_{i}}\right)
$$

where $g=9.81 \mathrm{~m} / \mathrm{s}^{2}$ is gravitational acceleration. In the above $h_{c}=0.25$ represents a so-called exposure factor (van Rijn, 2007), which accounts for larger particles being more exposed to the flow than smaller particles on graded sediment beds. Following van Rijn (2007), a default value $h_{c}=0.25$ is used, unless otherwise noted. Note that the effect of this parameter can be switched off simply by taking $h_{c}=0$.

\subsection{Suspended sediment model}

The hydrodynamic model is likewise coupled with a turbulent-diffusion based description for the simulation of the suspended sediment concentration (see e.g. Fredsøe and Deigaard, 1992, p. 238). The concentration representing each weight fraction is simulated individually according to:

$$
\frac{\partial c_{i}}{\partial t}=\frac{\partial\left(w_{s, i} c_{i}\right)}{\partial y}+\frac{\partial}{\partial y}\left(\epsilon_{s} \frac{\partial c_{i}}{\partial y}\right)
$$

where $w_{s, i}$ is the settling velocity of the $i$ th grain fraction, and $\epsilon_{s}=\beta_{s} \nu_{T}+\nu$ is the diffusion coefficient. The molecular viscosity is included here purely for numerical reasons. By default, the value $\beta_{s}=2$ is utilized herein (note that $\beta_{s}$ is the inverse of the Prandtl-Schmidt number discussed in other works); This value is in agreement with recent work conducted by e.g. Hsu and Liu (2004); Ruessink et al. (2009); Hassan and Ribberink (2010); Fuhrman et al. (2013). Eq. (19) is solved for $b \leq y \leq h_{m}$, where $b=2 d_{50}$ is taken the as the fixed reference level, and $h_{m}$ is the total height of the model domain. The calculation of the instantaneous rate of suspended sediment transport for each fraction is via

$$
q_{S, i}=\int_{b}^{h_{m}} u c_{i} d y
$$

Reference concentration boundary conditions are imposed at the lower $y=b$ level. Specifically, the reference concentration formula of Zyserman and Fredsøe (1994b) (altering the maximum concentration from 0.46 to 0.32 , as suggested by Zyserman and Fredsøe, 1994a) will be utilized, though in a slightly unconventional way: Rather than being based on the median grain size $d_{50}$, it will be assumed to be valid (on a weighted basis), for each individual weight fraction, yielding:

$$
c_{b 0, i}=w_{f, i} \frac{0.331\left(\theta_{i}-\theta_{c}\right)^{1.75}}{1+\frac{0.331}{0.32}\left(\theta_{i}-\theta_{c}\right)^{1.75}} .
$$

Note that this follows a similar approach as used by Li and Davies (2001), there making use of the reference concentration method of Engelund and Fredsøe (1976). When applied individually for each grain fraction $i$, it is likewise assumed that the concentrations computed from (21) will best represent those at the "natural" reference level for each grain size i.e. at $y=b_{i}=2 d_{i}$, rather than at the fixed (common) reference level $y=b=2 d_{50}$ used herein. To translate the concentrations from (21) to a common reference level (convenient, as it 

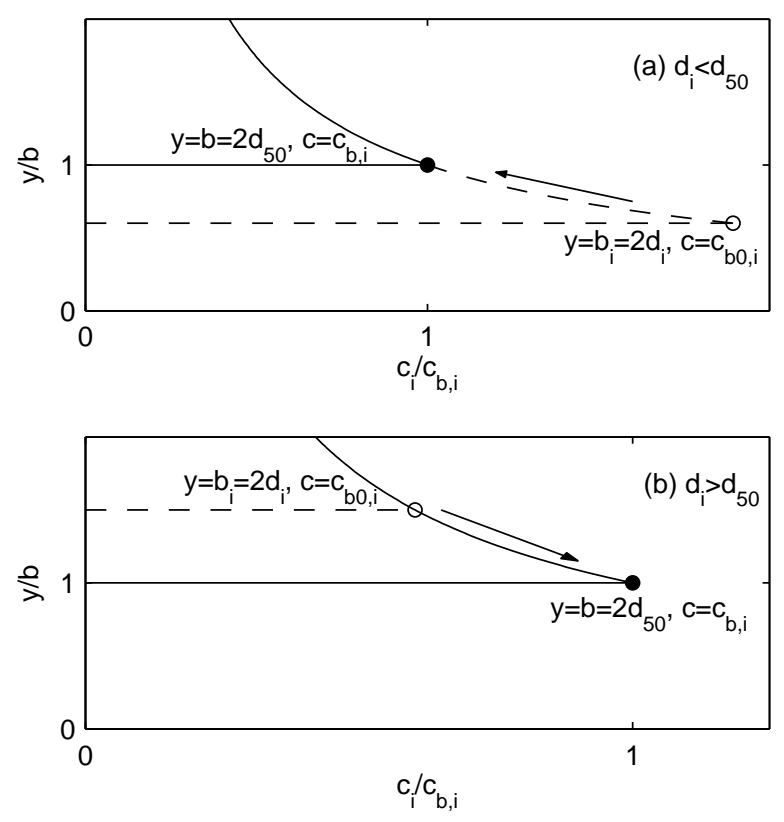

Figure 1: Demonstration of the reference concentration correction utilized in the present approach, resulting in (a) a decreased reference concentration $c_{b, i}$ when $d_{i}<d_{50}$ and (b) an increased reference concentration $c_{b, i}$ when $d_{i}>d_{50}$. The open circles represent the concentration at $y=b_{i}=2 d_{i}$ calculated via (21), whereas the filled circles represent the concentration utilized at the reference level $y=b=2 d_{50}$, after application of $(22)$.

enables use of a single computational mesh for suspended sediments of all fractions), we then introduce the following additional modification for application at $y=b$ :

$$
c_{b, i}=c_{b 0, i}\left(\frac{b_{i}}{b}\right)^{\Gamma}, \quad y=b .
$$

This adjustment is reasonable for moderately graded sediment distributions. As this modification accounts for the individual reference levels $b_{i}$ to be higher (lower) than $b$ for larger (smaller) grains, its inclusion will effectively increase (decrease) the amount of sediment put into suspension, relative e.g. to direct application of (21) at $y=b$. Both of these scenarios (i.e. $d_{i}<d_{50}$ and $d_{i}>d_{50}$ ) are demonstrated conceptually in Figure 1. A theoretical basis and further discussion of this modification is presented in the Appendix, where it is demonstrated that the parameter $\Gamma$ is, in fact, linked to the Rouse parameter for steady flows. For simplicity, however, a default fixed value $\Gamma=1.25$ will be used in the forthcoming unsteady simulations. The effect of switching this feature off will also be considered in what follows, achieved simply by taking $\Gamma=0$, yielding $c_{b, i}=c_{b 0, i}$.

It is well known that at high concentrations the settling velocity becomes reduced, compared to the settling velocities of individual grains (e.g. Fredsøe and Deigaard, 1992). In the current sediment transport model these "hindered settling" effects are taken into account by allowing the settling velocity $w_{s, i}$ for each fraction to depend on the local total 
suspended sediment concentration according to Richardson and Zaki (1954):

$$
w_{s, i}=w_{s 0, i}(1-c)^{n_{i}}
$$

where the exponent $n_{i}$ is computed for the $i$ th fraction according to

$$
\begin{array}{ccl}
n_{i}=4.35 R_{i}^{-0.03}, & 0.2<R_{i}<1, \\
n_{i}=4.45 R_{i}^{-0.1}, & 1<R_{i}<500, \\
n_{i}= & 2.39, & R_{i}>500 .
\end{array}
$$

The settling Reynolds number is defined as $R_{i}=w_{s 0, i} d_{i} / \nu$. The base settling velocity for the $i$ th fraction $w_{s 0, i}$ is calculated empirically based on the drag coefficient methodology presented in Fredsøe and Deigaard (1992), making use of the explicit solution method described in Fuhrman et al. (2013).

Finally, to prevent forcing $c_{b, i}=0$ during periods of flow reversal, the actual $c_{b, i}$ value retained during simulations are taken as the maximum of those computed from the reference concentration above and those extrapolated from the two points nearest the bed. This prevents un-physical "overloading" conditions (i.e. where reference $c_{b, i}$ is forced to be smaller than the concentration immediately above) from ever occurring in the model.

The model described above, again, represents a multiple grain fraction extension of the original "MatRANS" model presented by Fuhrman et al. (2013), which was limited to a single uniform sediment size $d$. The numerical model is implemented within the Matlab environment, making use of finite difference approximations for computing vertical derivatives, with time integration based on Matlab's ode15s solver. Note that the model also incorporates other potential capabilities not specifically utilized within the present study, including the ability to simulate e.g. boundary layer streaming (and other convective term) effects (Fuhrman et al., 2013) as well as e.g. laminar-to-turbulence transition (Williams and Fuhrman, 2016). The authors are happy to make the code (composed primarily of two Matlab M-files) freely available to others, e.g. for research or educational purposes, upon request to the corresponding author.

\section{Sediment transport beneath skewed waves}

\subsection{Model validation}

In this section we will validate the full model against two recent experimental data sets (O'Donoghue and Wright, 2004a; Hassan and Ribberink, 2005) involving graded sediment transport beneath skewed waves on flat beds within oscillating tunnel facilities. O'Donoghue and Wright (2004a) performed a series of 12 tests, 6 of which utilized well-sorted sediments (fine sand with $d=0.15 \mathrm{~mm}$, medium sand with $d=0.28 \mathrm{~mm}$, and coarse sand with $d=0.51$ $\mathrm{mm}$ ), and 6 of which utilized the three sediment mixtures (Mix X1, Mix X2, and Mix X3, having $d_{50}=0.19 \mathrm{~mm}, 0.28 \mathrm{~mm}$, and $0.28 \mathrm{~mm}$, respectively) as indicated in Table 1 . Two different wave periods $T=5 \mathrm{~s}$ and $T=7.5 \mathrm{~s}$ were likewise considered, in combination with free stream velocities of the form (12), with $U_{1 m}=1.21 \mathrm{~m} / \mathrm{s}$ and $U_{2 m}=0.31 \mathrm{~m} / \mathrm{s}$. The shape of the velocity-skewed signal is provided in Figure 2. For the present validation, we will consider all 12 of their experimental conditions, hence demonstrating model performance 
Table 1: Mixture descriptions for O'Donoghue and Wright (2004a).

\begin{tabular}{cccc}
\hline Mix & $d_{50}(\mathrm{~mm})$ & grain diameters $(\mathrm{mm})$ & weight fractions \\
\hline $\mathrm{X} 1$ & 0.19 & {$[0.15,0.28,0.51]$} & {$[0.60,0.30,0.10]$} \\
$\mathrm{X} 2$ & 0.28 & {$[0.15,0.28,0.51]$} & {$[0.20,0.60,0.20]$} \\
$\mathrm{X} 4$ & 0.28 & {$[0.15,0.51]$} & {$[0.50,0.50]$} \\
\hline
\end{tabular}

Table 2: Mixture descriptions for Hassan and Ribberink (2005).

\begin{tabular}{cccc}
\hline Mix & $d_{50}(\mathrm{~mm})$ & grain diameters $(\mathrm{mm})$ & weight fractions \\
\hline $\mathrm{K}$ & 0.194 & {$[0.13,0.34]$} & {$[0.50,0.50]$} \\
$\mathrm{P}$ & 0.24 & {$[0.21,0.97]$} & {$[0.70,0.30]$} \\
$\mathrm{S}$ & 0.15 & {$[0.13,0.34,0.97]$} & {$[0.60,0.20,0.20]$} \\
\hline
\end{tabular}

for both graded and well-sorted (uniform) sediments. Comparisons will be limited to the reported period-averaged total transport rates in what follows.

Hassan and Ribberink (2005) have likewise conducted a series of oscillating tunnel experiments involving sheet flow beneath skewed free-stream velocity signals of the form (12). Comparison will therefore similarly be made against their 19 pure wave cases involving uniform sands (grain diameters ranging from $d=0.13 \mathrm{~mm}$ to $0.97 \mathrm{~mm}$ ), corresponding to their $\mathrm{B}, \mathrm{C}, \mathrm{D}, \mathrm{R}$, and $\mathrm{Q}$ series (full period results only). These conditions consider wave periods ranging from $T=5 \mathrm{~s}$ to $12 \mathrm{~s}$. Additionally, we will consider their $\mathrm{K}, \mathrm{P}$, and $\mathrm{S}$ series of tests (a total of 10 cases) utilizing the sediment mixtures indicated in Table 2. For each of their conditions the model is set up as described previously, with the velocity magnitudes $U_{1 m}$ and $U_{2 m}$ set in accordance with the reported values for each case. In addition to the total period-averaged transport rate for the sediment mixtures, Hassan and Ribberink (2005) also report the period-averaged transport for each weight fraction. Hence, comparison with our model results will be attempted for each of these quantities in what follows, i.e. transport rates both in terms of the total transport, as well as that for each individual weight fraction.

For all tests considered, the model depth is set to $h_{m}=0.25 \mathrm{~m}$, corresponding to half of the distance from the undisturbed sand bottom to the roof of the experimental tunnel. The experiments on the sand beds were typically carried out over 25 flow cycles.

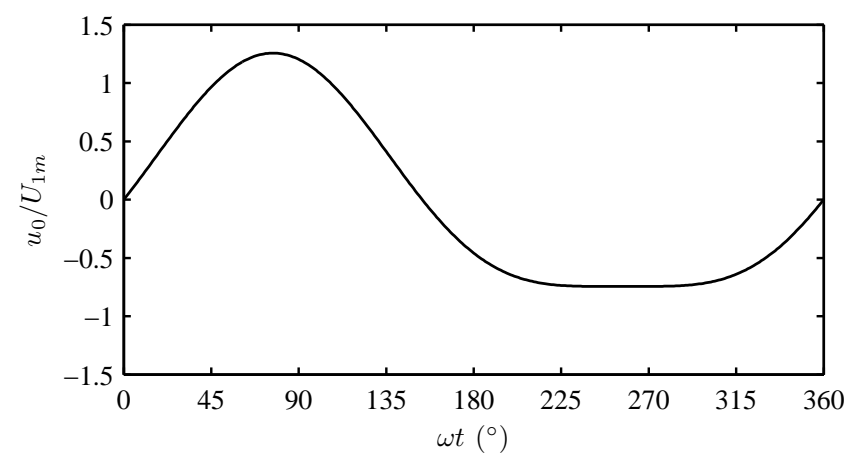

Figure 2: Free stream velocity signal from the experimental study of O'Donoghue and Wright (2004b). 
Hence, for comparison all computed results presented will be taken from the 12th cycle i.e. corresponding approximately to the middle of the experimental duration. This ensures that the boundary layer within the model domain will be at a similar stage of development as in the experiments. This protocol follows that of Fuhrman et al. (2013). It is important to note that that, beyond the first few cycles, the predicted net transport rates vary little (of the order 10\%) over the full coarse of the experimental duration, so the precise cycle considered is of little significance.

Model validation results for the experimental conditions described above are depicted in Figure 3. In this comparison, all model features are switched on, making use of the previously-indicated default parameters: $\mu_{d}=0.65, \beta_{s}=2.0, \Gamma=1.25$ and $h_{c}=0.25$. In this figure, and in many that follow, we will maintain the following organization: Subplot $(a)$ presents comparison of computed and measured period-averaged transport rates (combined bed and suspended load) for individual weight fractions (characterized by their respective diameters $d_{i}$ ), as reported by Hassan and Ribberink (2005); Subplot (b) presents comparison of the total period-averaged transport of sediment mixtures, as reported by Hassan and Ribberink (2005) and O'Donoghue and Wright (2004b); Finally, subplot (c) presents a comparison of the total transport rate for experiments involving uniform sediments, as reported by Hassan and Ribberink (2005) and O'Donoghue and Wright (2004b). Note that in this, and in selected subsequent figures, we utilize the abbreviations $\mathrm{HR}$ and $\mathrm{OW}$, respectively, for Hassan and Ribberink (2005) and O'Donoghue and Wright (2004b). On each subplot, the full line represents the line of perfect agreement, whereas the region between the dashed lines represents agreement within plus or minus a factor of two, often taken as acceptably accurate when making sediment transport predictions.

We will first discuss the comparison of transport rates for the individual grain fractions of Hassan and Ribberink (2005), again presented in Figure 3a. Despite the great difficulty in simultaneously predicting the transport of a wide range of sediment sizes (from fine to course sands), the results appear reasonable, with most of the predicted net transport rates being within a factor of two of those measured. In some cases, due to phase lag effects, the finest sediments were in fact transported backwards; While the magnitude of negative transport rates for these fine sand fractions tend to be over-predicted by the model in such cases, this phenomenon is at least qualitatively captured. There appears to be a slight tendency towards the over-prediction of transport rates for the finer fractions, and under-prediction for coarser fractions, though exceptions are apparent for each.

The general quality of predicting the total sediment transport rates for the mixtures considered can be further seen in Figure 3b, now considering both the OW and HR experiments. The HR cases, which contain the widest range of grain sizes of the two, are seen to consistently lie very near the line of perfect agreement. The clustering for the OW mixture experiments is less impressive, but is still acceptable, with all but two cases being with the factor two (dashed lines). There does also not appear to be any consistent or systematic trend towards either over- or under-predicting the total transport rate for sediment mixtures based on these data sets, as the clustering is approximately centered about the line of perfect agreement. All predicted net transports for the mixtures are positive, in line with the experiments.

The excellent agreement with the Hassan and Ribberink (2005) data set is further demonstrated in Figure 4, which depicts the total net transport rate versus the third mo- 

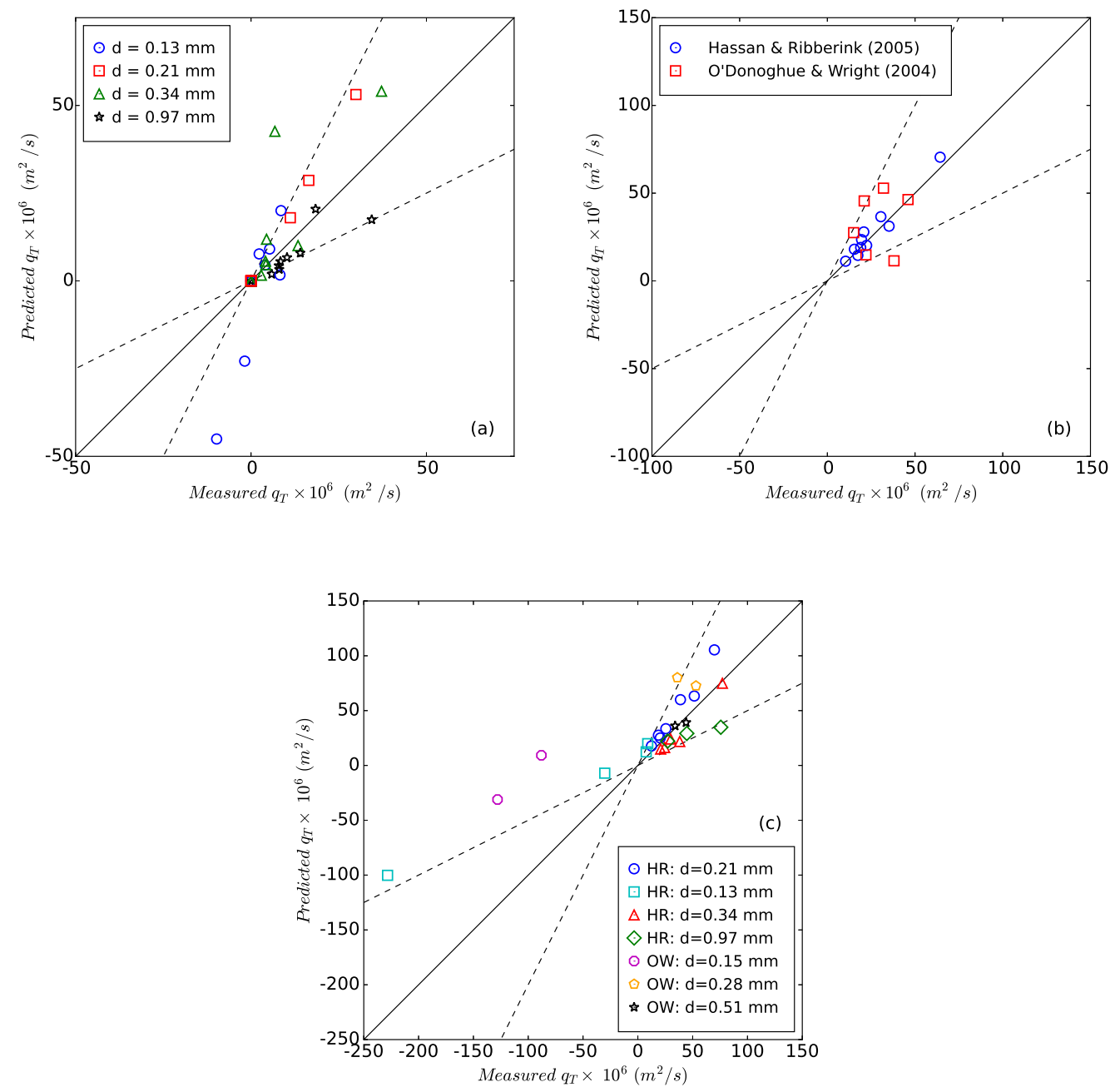

Figure 3: Summary of measured versus predicted (full model) period-averaged sediment transport rates, based on skewed-wave experiments of HR: Hassan and Ribberink (2005) and OW: O'Donoghue and Wright (2004b). Sub-plot (a) compares transport rates for individual weight fractions (from HR), (b) compares total transport rates of mixtures (both $\mathrm{HR}$ and $\mathrm{OW}$ ), and (c) compares total transport rates for uniform sands (both HR and OW). 


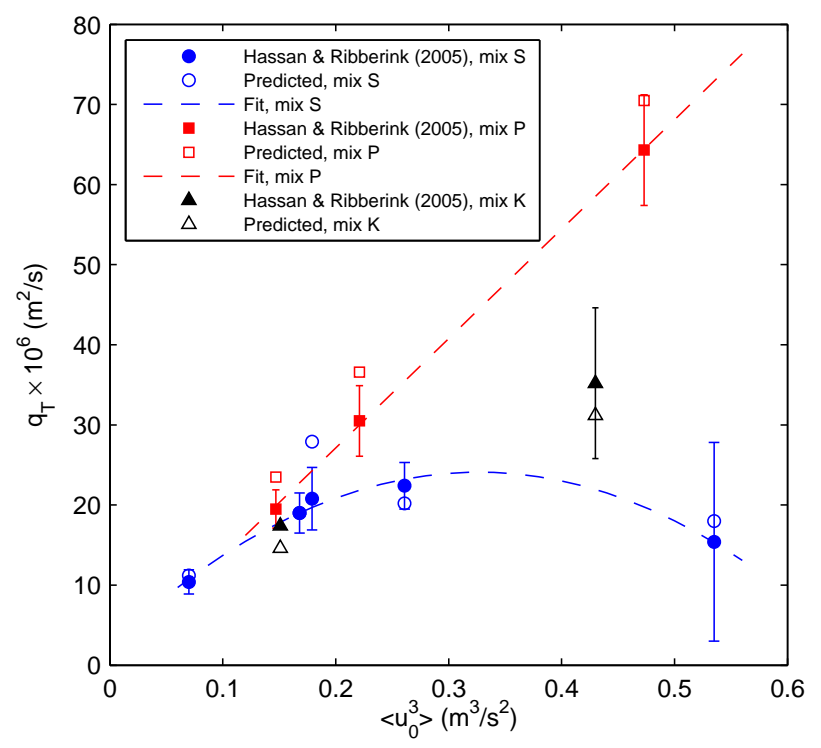

Figure 4: Measured and predicted net sediment transport rates versus the third moment of the free stream velocity $\left\langle u_{0}^{3}\right\rangle$ for the non-uniform sand cases from Hassan and Ribberink (2010). The experimental error bars for each case are also shown. The dashed lines are curve fits to the experimental data from mix $\mathrm{S}$ and P.

ment of the free stream velocity $\left\langle u_{0}^{3}\right\rangle$ for all of their non-uniform sand cases. The present model clearly captures e.g. the linear growth in the net transport for mix P (containing $d=0.21 \mathrm{~mm}$ and $0.97 \mathrm{~mm}$ ). The model likewise captures e.g. the deviation from such a linear trend for larger $\left\langle u_{0}^{3}\right\rangle$ with mix S (mixture containing $d=0.13 \mathrm{~mm}, 0.34$ and $0.97 \mathrm{~mm}$ ). Inspection of the model results indicates that the break in this linear trend for mix $\mathrm{S}$ is due to unsteady effects of the fine sand fraction $(d=0.13 \mathrm{~mm})$, which is actually transported in the negative direction due to phase lag effects (this fraction fails to completely settle prior to flow reversal), as described previously. These effects were also speculated directly by Hassan and Ribberink (2005).

As final validation, we compare computed sediment transport rates with those measured for the uniform (well-sorted) sediment beds in Figure 3c. This is an important test, as cases involving uniform sized sediments should obviously be considered as special cases of those involving graded sediments. As seen, the model also makes reasonable predictions in these cases. For the cases involving positive transport, all but two of the predicted transport rates are within a factor of two of those measured (namely one from OW, with $d=0.28 \mathrm{~mm}$ and one from HW, with $d=0.97 \mathrm{~mm}$ ). For the fine sand cases demonstrating net negative transport rates (again due to phase lag effects), the model under-estimates the transport magnitude, but again captures this phenomenon qualitatively in three of the four cases.

Regarding the importance of phase-lag effects beneath velocity-skewed flows, it can be noted that recent numerical results of Fuhrman et al. (2013) and Kranenburg et al. (2013) have revealed that inclusion of progressive wave streaming and other convective term effects can, in fact, "re-reverse" the transport of fine grained sediments in such cases to be in the positive direction. Hence, based on their results, it seems that the phenomenon of negative 


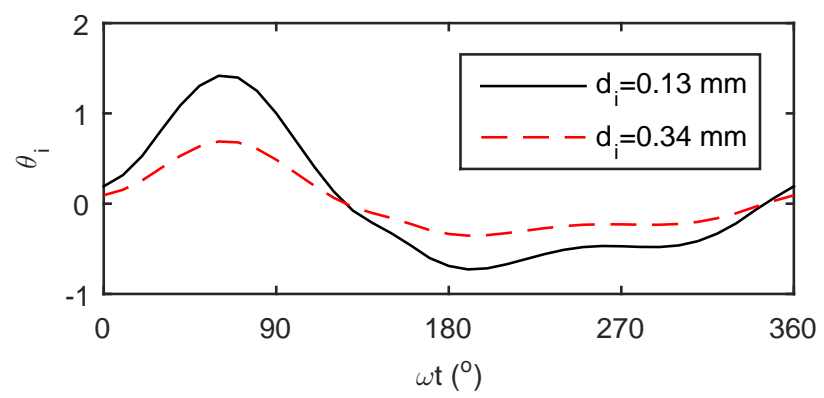

Figure 5: Computed time variation of the Shields parameters for case K2 of Hassan and Ribberink (2010) using default parameters.

transport of fine sands beneath skewed wave signals may simply be an experimental artifact caused by the streamwise-uniform nature of the flow within oscillating tunnel facilities.

In summary, the results depicted in Figures 3 and 4 collectively demonstrate the ability of the present model to predict period-averaged wave-induced sediment transport rates for sediment mixtures, involving a wide range of particle sizes (i.e. ranging from fine to coarse sands). This is true in terms of the transport of individual size fractions within mixtures (Figure $3 a$ ), as well in terms of the total transport of all sizes within said mixtures (Figure $3 b$ and 4 ). Comparisons also demonstrate that the model maintains reasonable accuracy for predicting wave-induced sediment transport rates at the limit of uniform sediment grains (Figure $3 c$ ) over a similar range in sediment sizes as comprising the considered mixtures.

\subsection{Influence of individual parameters}

Having validated the full model in the previous sub-section for predicting wave-induced transport of graded and uniform sediments beneath skewed waves, we will now investigate the sensitivity in the predictive capabilities to changes in several of the parameters utilized, with the hope of clarifying their importance, or in some cases, the relative lack thereof. For this purpose, we will systematically repeat the comparisons made in Figure 3, but with a single one of our default parameters: $\mu_{d}=0.65, \beta_{s}=2, \Gamma=1.25$ and $h_{c}=0.25$ changed in isolation.

To also aid in the forthcoming discussion, we will present selected temporal variations from a single case (corresponding to case K2 of Hassan and Ribberink, 2010). This case has been selected for demonstration purposes, as it involves intermediate sediment $\left(d_{50}=0.194\right.$ $\mathrm{mm})$ and flow characteristics $\left(T=6.5 \mathrm{~s}, U_{1 m}=0.83 \mathrm{~m} / \mathrm{s}, U_{2 m}=0.28 \mathrm{~m} / \mathrm{s}\right)$, and is comprised of only two weight fractions (one larger than and one smaller than $d_{50}$, see Table 2 ) for simplicity. The computed time variations of the Shields parameters $\theta_{i}$ for this case (using default parameters) are depicted in Figure 5. Similarly, time series of computed bed load $q_{B, i}$ and suspended load $q_{S, i}$ transport rates are depicted, respectively, in Figures 6 and 7 for selected parameter settings. Finally, the computed concentration profiles $c_{i}$ at the instant when the free stream flow is maximum for this case are plotted in Figure 8, again for selected parameter settings. In Figures 6, 7, and 8 the full lines depict results using the default model parameters, which will serve as a basis for comparison.

To begin this investigation, we will consider the comparison with the coefficient of dynamic friction changed from 0.65 to $\mu_{d}=1$. The resulting comparison of predicted 
(a) $d_{i}=0.13 \mathrm{~mm}$

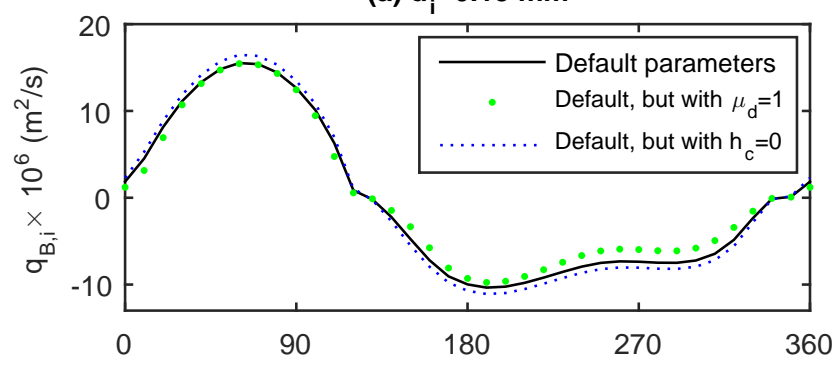

(b) $d_{i}=0.34 \mathrm{~mm}$

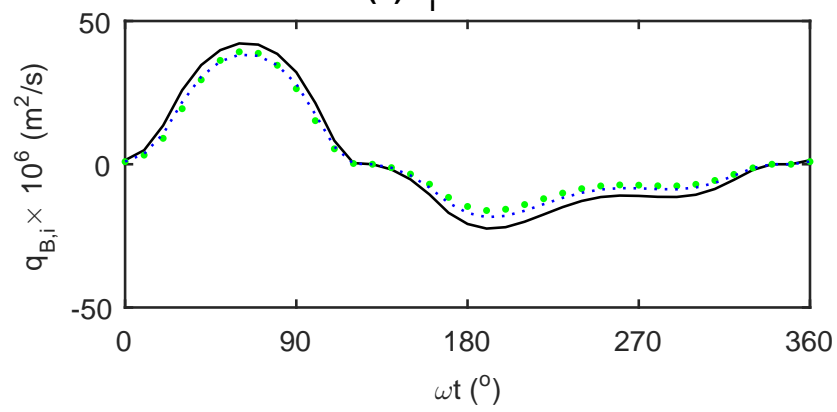

Figure 6: Computed time variation of bed load transport for case K2 of Hassan and Ribberink (2010).

(a) $d_{i}=0.13 \mathrm{~mm}$

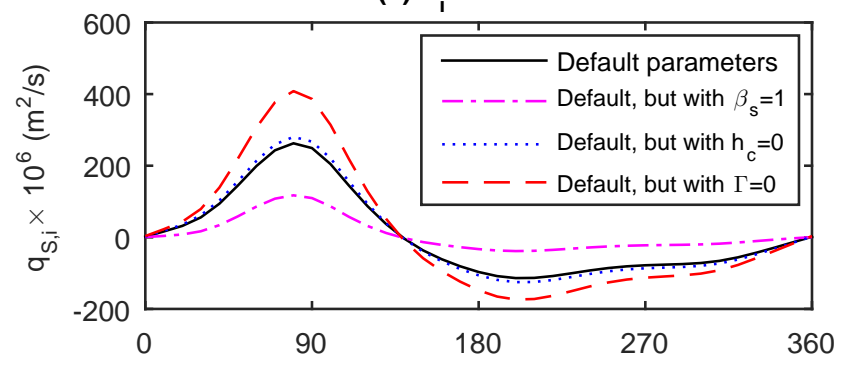

(b) $d_{i}=0.34 \mathrm{~mm}$

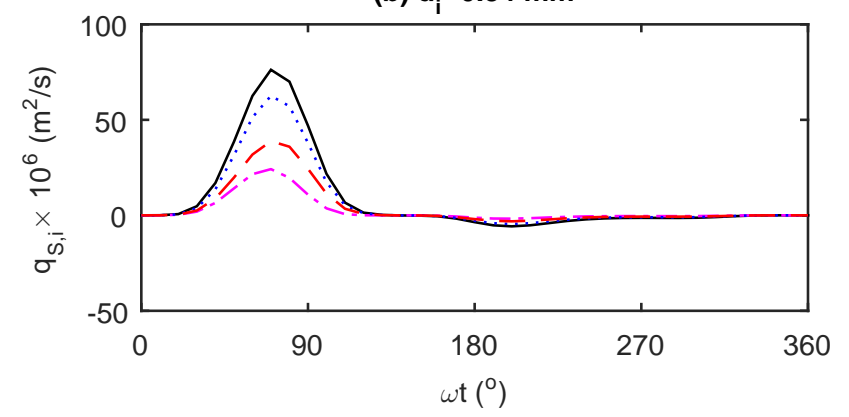

Figure 7: Computed time variation of suspended load transport for case K2 of Hassan and Ribberink (2010). 
(a) $d_{i}=0.13 \mathrm{~mm}$

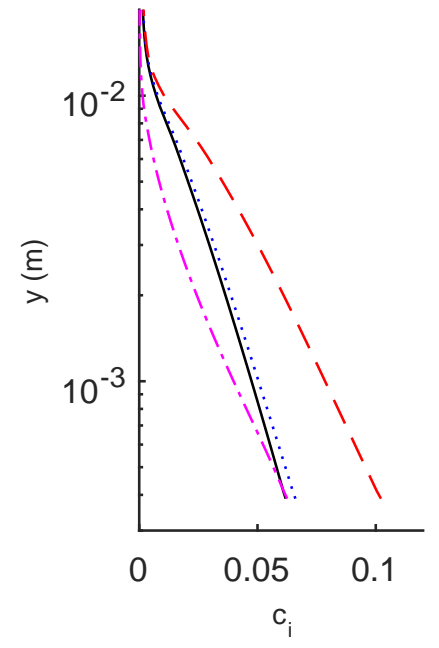

(b) $d_{i}=0.34 \mathrm{~mm}$

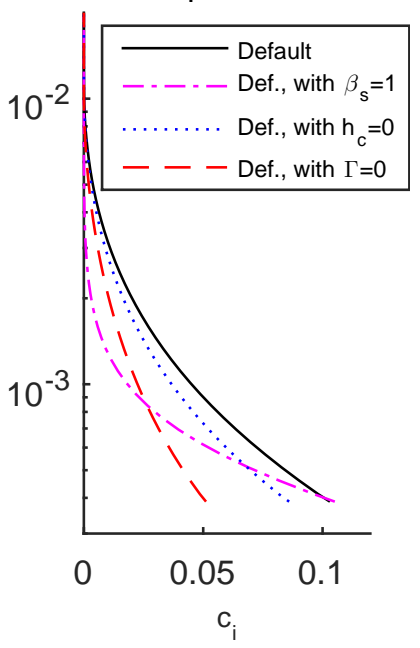

Figure 8: Computed suspended sediment concentration profiles at peak flow velocity for case K2 of Hassan and Ribberink (2010).

versus measured period-averaged sediment transport rates are presented in Figure 9, in an analogous fashion to Figure 3. As seen here, changing the parameter $\mu_{d}$ has relatively little effect on the total predicted sediment transport, as the resulting plots bear close resemblance to those in Figure 3 . Within the present formulation, modifying $\mu_{d}$ only effects the weighted probability of moving grains $p_{i}$ in (17), which in turn effects the predicted bed load transport $q_{B, i}$ from (16). For small Shields parameters near critical, the increase in $\mu_{d}$ may reduce bed load transport rates by as much as $45 \%$. In the cases considered herein, however, the sediment transport is generally within the sheet flow regime, where the Shields parameters are relatively large, and computed suspended sediments comprise a far greater percentage of the total transport (in RANS based models such as used herein, the dynamics of the sheet flow layer are largely represented within the suspended sediment model, as illustrated previously e.g. in Fuhrman et al., 2013). Hence, while modifying $\mu_{d}$ may have a greater effect in cases where computed bed load is more dominant, it does not affect the present results significantly. This is further illustrated by the temporal evolution of the bed load transport rates $q_{B, i}$ with $\mu_{d}=1$ (shown as dots) depicted on Figure 6. Consistent with the above description, the reduction (relative to the default settings, full lines) in the transport rate is mild, and is most apparent when the Shields parameters are relatively low i.e. for the larger grain fraction (Figure 6b) and during trough regions of the flow.

Next we will consider the effect of modifying the sediment diffusivity factor from our default value of $\beta_{s}=2$ to a reduced value of $\beta_{s}=1$. The resulting period-averaged transport rates are presented in Figure 10, which can again be directly compared with Figure 3 . This parameter affects the diffusion coefficient within (19), and hence has a direct effect on the simulated suspended sediment via the turbulent diffusion equations. This parameter, therefore, has an important effect in overall sediment transport predictions in the sheet flow regime. As is apparent in Figure 10, reducing $\beta_{s}$ generally results in the systematic 

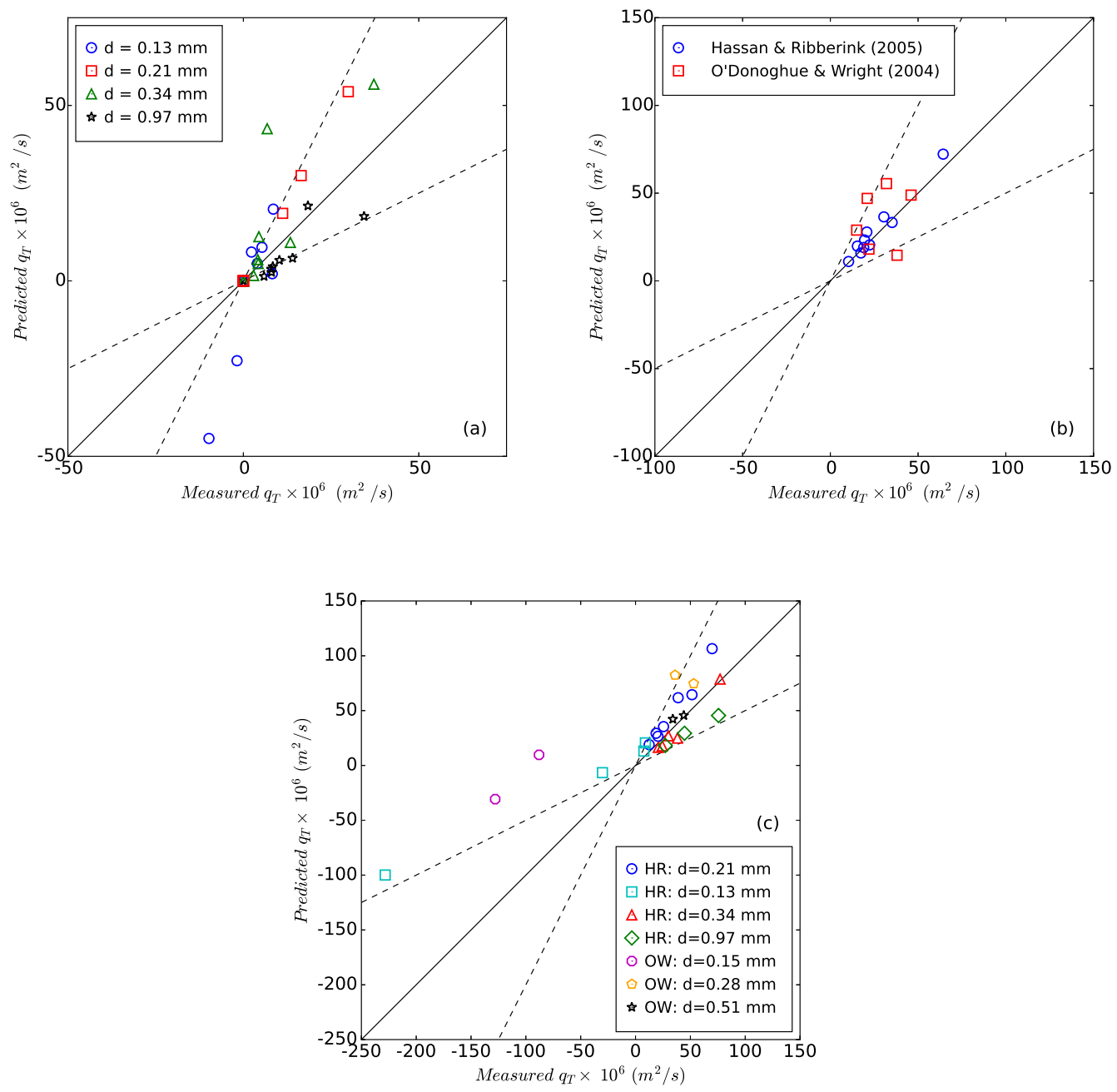

Figure 9: Summary of measured versus predicted (using $\mu_{d}=1.0$ ) period-averaged sediment transport rates, based on skewed-wave experiments of HR: Hassan and Ribberink (2005) and OW: O'Donoghue and Wright (2004b). Sub-plot (a) compares transport rates for individual weight fractions (from HR), (b) compares total transport rates of mixtures (both $\mathrm{HR}$ and $\mathrm{OW}$ ), and (c) compares total transport rates for uniform sands (both HR and OW). 
under-prediction of total wave-induced transport. This is readily apparent, both in the total transport of graded (Figure 10b) and uniform sediments (Figure 10c). A comparison of the fine sand results in Figure $10 a$ and $10 c$ likewise demonstrates that phase lag effects are not well accounted for, as negative transport rates for fine grains are generally no longer even qualitatively predicted. This comparison hence supports utilizing $\beta_{s}=2$ within the present formulation, which is in line with similar findings for models limited to uniform sediments e.g. Hsu and Liu (2004), Ruessink et al. (2009), Hassan and Ribberink (2010), Fuhrman et al. (2013), and Kranenburg et al. (2013). The dramatic reduction in transport rates described above is likewise apparent in the temporal evolutions of $q_{S, i}$ with $\beta_{s}=1$ shown in Figure 7 (dashed-dotted lines), when compared with the default setting (full lines, with $\beta_{s}=2$ ). The effect is also very clear in the vertical suspended sediment concentration profiles, Figure 8. The reduction is very apparent with both weight fractions comprising the mixture.

We will now investigate the effects of switching off the so-called exposure factor, which can conveniently be accomplished simply by setting $h_{c}=0$. The resulting period-averaged transport rates are depicted in Figure 11. It can be noted that there are only two subplots on this figure. This is because the exposure factor only affects cases involving graded sediments, hence the uniform sediment results are identical to Figure $3 c$, and are thus not repeated. Comparing the results shown in Figure 11 with those shown in Figure $3 a, b$, it is evident that switching off this parameter has a detrimental effect in the predicted transport rates for the larger grains, which makes sense as this parameter is designed to account for their increased exposure. As this parameter is applied directly onto the effective Shields parameter, it inflates both the bed load transport rates as well as the reference concentrations. For the finer grains, the exposure factor does not have strong influence, as the factor $d_{i} / d_{50}$ is much closer to unity for these fractions. The temporal variations in the transport rates with the exposure factor switched off $\left(h_{c}=0\right)$ are likewise depicted in Figures 6 and 7 (dotted lines); see also the respective concentration profiles in Figure 8. Consistent with the description above, switching off the exposure factor is seen to reduce the collective transport of the larger grain fraction.

We will finally investigate the effects of switching off the reference concentration modification (22), again achieved simply by setting $\Gamma=0$. As before, the uniform sediment results under this setting are identical to Figure $3 c$, and are not shown to prevent redundancy. The resulting transport rates for the graded mixtures are shown in Figure 12. As seen there, switching off this modification leads to systematic under-prediction of the transport rates for the larger grain fractions, i.e. $d=0.34 \mathrm{~mm}$ and $d=0.97 \mathrm{~mm}$ in Figure $12 a$. The resulting total net transport rates for the graded sediments, Figure 12b, are considerably worse than when this modification is included, Figure $3 b$, with several of the results now yielding the incorrect sign of the net transport. The net effect of this modification is to increase (decrease) the coarser (finer) fractions put into suspension, which is also clear from comparison of the temporal evolutions depicted in Figure 7, as well as the vertical concentration profiles, Figure 8. Based on the present results, this modification significantly helps remedy an otherwise systematic tendency towards over- (under-) predicting the transport rates of fine (coarse) grain fractions.

Based on the comparisons made in this section, it is clear that certain parameters effect the predictive skill of the present model more than others. The best results achieved turn out 

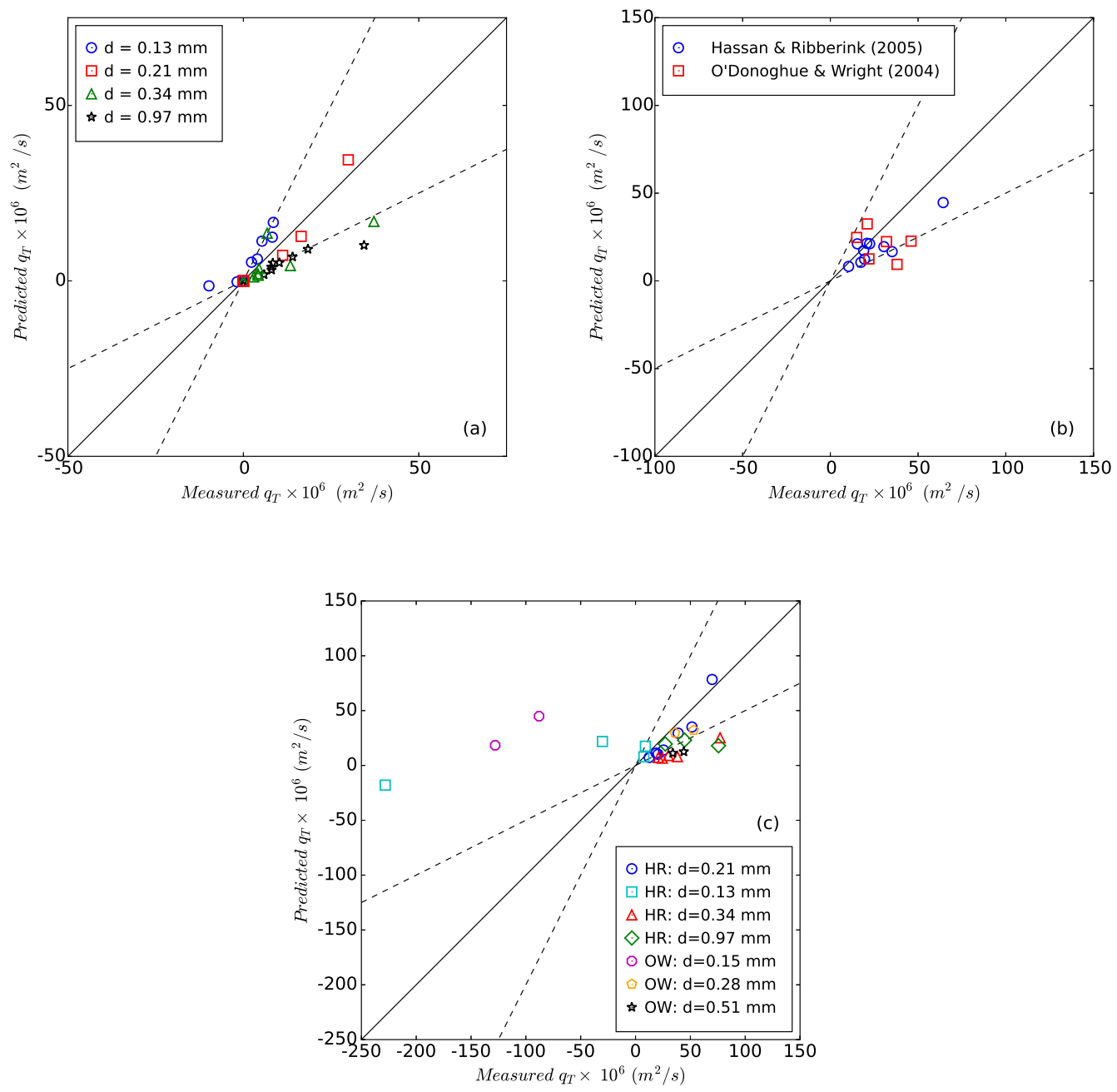

Figure 10: Summary of measured versus predicted (using $\beta_{s}=1.0$ ) period-averaged sediment transport rates, based on skewed-wave experiments of HR: Hassan and Ribberink (2005) and OW: O'Donoghue and Wright (2004b). Sub-plot (a) compares transport rates for individual weight fractions (from HR), (b) compares total transport rates of mixtures (both $\mathrm{HR}$ and $\mathrm{OW}$ ), and (c) compares total transport rates for uniform sands (both HR and OW). 

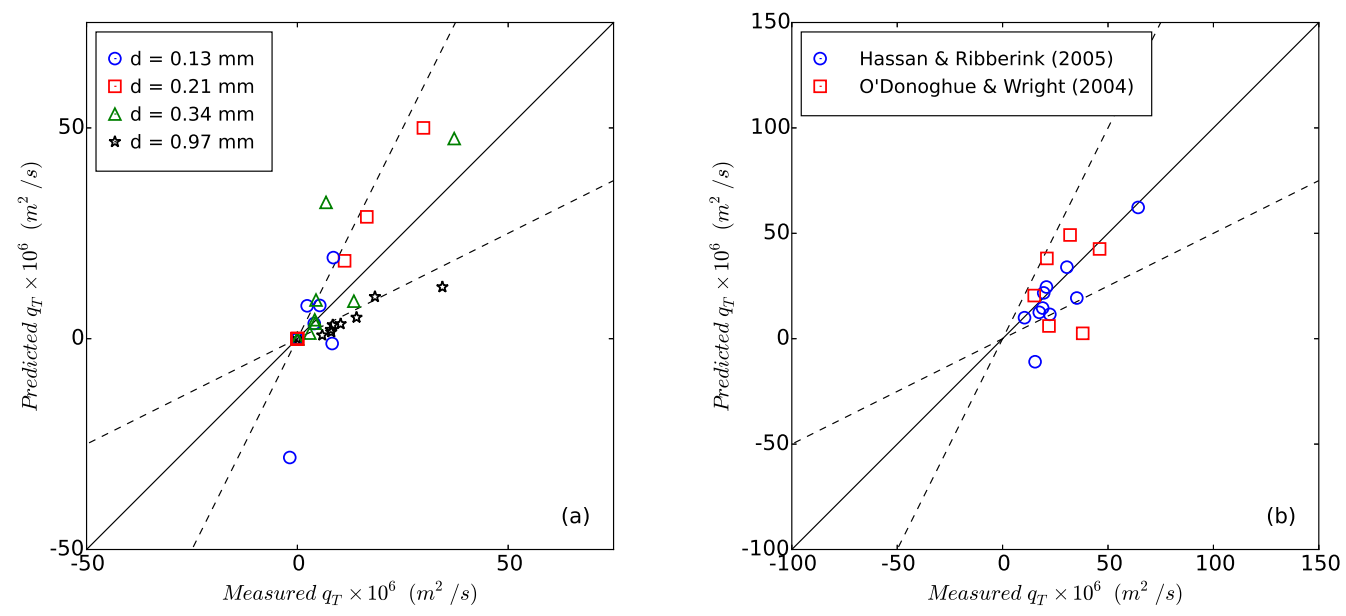

Figure 11: Summary of measured versus predicted (using $h_{c}=0$ ) period-averaged sediment transport rates, based on skewed-wave experiments of HR: Hassan and Ribberink (2005) and OW: O'Donoghue and Wright (2004b). Sub-plot (a) compares transport rates for individual weight fractions (from HR), while (b) compares total transport rates of mixtures (both HR and OW).
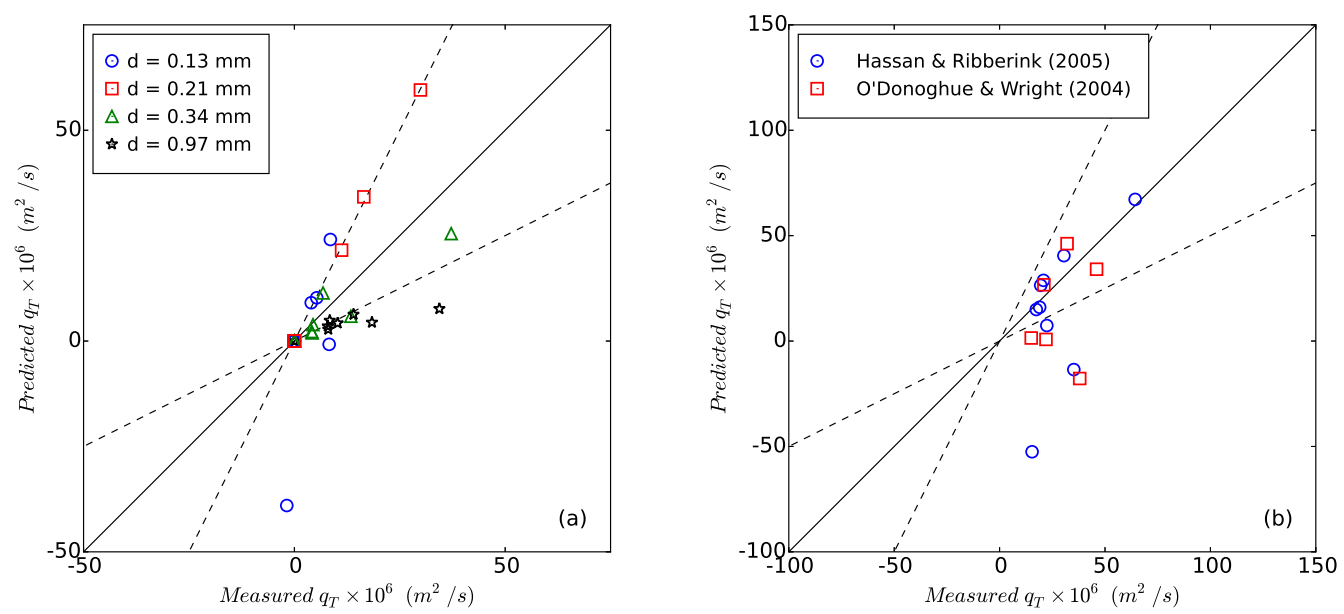

Figure 12: Summary of measured versus predicted (using $\Gamma=0$ ) period-averaged sediment transport rates, based on skewed-wave experiments of HR: Hassan and Ribberink (2005) and OW: O'Donoghue and Wright (2004b). Sub-plot (a) compares transport rates for individual weight fractions (from HR), while (b) compares total transport rates of mixtures (both $\mathrm{HR}$ and $\mathrm{OW}$ ). 
to be with the default settings selected for the model validation, Figure 3 , with $\mu_{d}=0.65$, $\beta_{s}=2, h_{c}=0.25$, and $\Gamma=1.25$. This is encouraging, especially given that the latter two coefficients account for physical effects associated with 1) greater exposure of coarse grains, and 2) adjusting the individual reference concentrations for application at the (shared) fixed reference level. Of the two, the effect of the latter is the most pronounced. As the (sheet flow regime) cases considered here are largely dominated by suspended sediment transport, the effects of changing $\mu_{d}$ are not very pronounced. Utilizing an optimal value for $\beta_{s}=2$ turns out to be quite important, as reduction to $\beta_{s}=1$ results in poor predictions even at the uniform grain size limit.

\section{Sediment transport beneath asymmetric waves}

\subsection{Uniform sediments}

The previous section has considered in depth simulations involving the transport of both graded and non-graded sediments beneath velocity-skewed wave signals, demonstrating acceptable accuracy in both cases. In practice, however, nonlinear (near bottom) wave signals may be either skewed or front-back asymmetric (or some combination of the two, depending e.g. on the wave nonlinearity, beach slope, and location i.e. inside or outside the surf zone). In this section we therefore consider similar model simulations involving the sediment transport induced by asymmetric waves, also commonly called "accelerationskewed" signals in the literature. To the authors' knowledge, data sets akin to those of O'Donoghue and Wright (2004b) and Hassan and Ribberink (2005) for the transport of graded sediments beneath asymmetric waves are unfortunately lacking in the literature. However, van der A et al. (2010) have recently conducted a series of 35 tests in an oscillating tunnel involving the sheet-flow transport of uniform sediments due to acceleration-skewed signals. Their tests considered well-sorted sediments with diameters $d=0.15 \mathrm{~mm}, 0.27 \mathrm{~mm}$, and $0.46 \mathrm{~mm}$, with wave periods $5 \mathrm{~s} \leq T \leq 9 \mathrm{~s}$, velocity magnitudes spanning approximately $0.84 \mathrm{~m} / \mathrm{s} \leq U_{w} \leq 1.30 \mathrm{~m} / \mathrm{s}$, with so-called acceleration skewness coefficients spanning $0.56 \leq \beta \leq 0.78$. In the present paper, we will compare specifically with their experimental results with $d=0.15 \mathrm{~mm}$ (fine sand) and $d=0.27 \mathrm{~mm}$ (medium sand). These results will likewise serve as reference for additional model simulations involving identical flows, but simulated with sediment mixtures having similar $d_{50}$ values, in the following sub-section. In addition, for completeness, we will likewise make similar comparisons against the 12 experimental results of Watanabe and Sato (2004) included in the SANTOSS dataset (van der Werf et al., 2009), involving sheet-flow sediment transport of well-sorted sands with $d=0.2 \mathrm{~mm}$ beneath acceleration-skewed flows having $T=5 \mathrm{~s}, 0.86 \mathrm{~m} / \mathrm{s} \leq U_{w} \leq 1.45 \mathrm{~m} / \mathrm{s}$, and $0.6 \leq \beta \leq 0.8$.

In all forthcoming results, the model is set-up and results analyzed in precisely the same fashion as described previously. The model is run exclusively with the default parameters: $\mu_{d}=0.65, \beta_{s}=2, \Gamma=1.25$ and $h_{c}=0.25$, hence incorporating all considered gradation effects. Following previous simulations of these uniform cases by Fuhrman et al. (2013), the driving pressure gradient is based on Eq. 15 with $\phi=0$ (sawtooth shaped flows), with the shape parameter $r$ set to yield the precise acceleration skewness coefficient $\beta$ reported by van der A et al. (2010), based on Eq. (14) of Abreu et al. (2010). Similarly, for the 


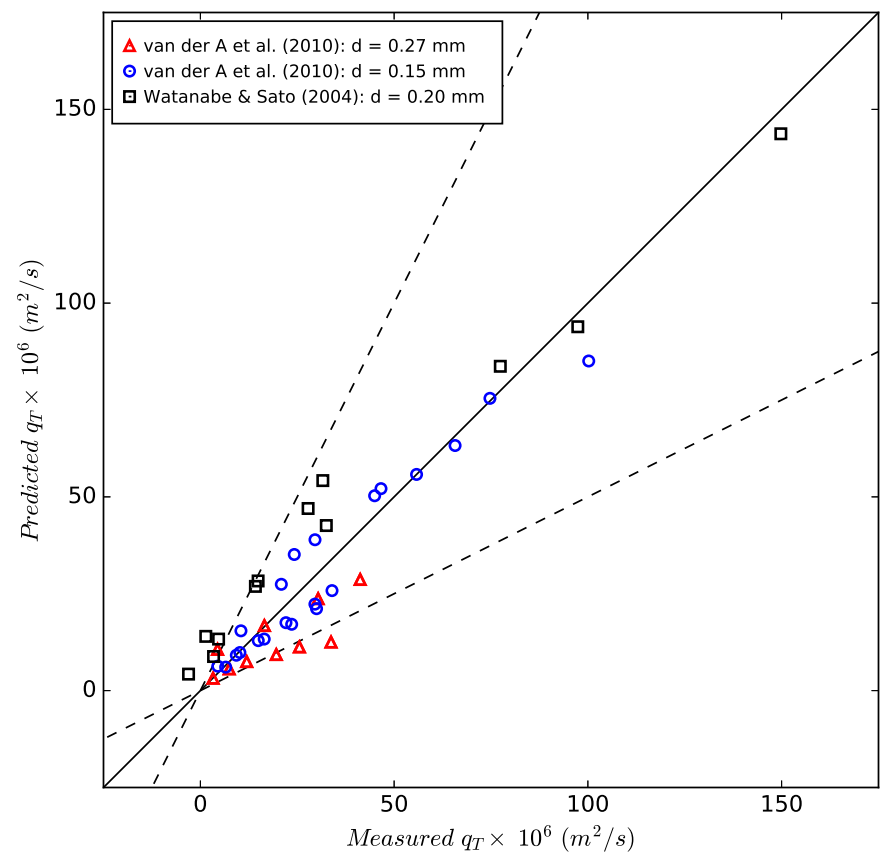

Figure 13: Summary of measured (van der A et al., 2010) and predicted period-averaged sediment transport rates for cases involving asymmetric waves.

experiments of Watanabe and Sato (2004) we use $\beta$ as directly reported in the SANTOSS dataset of van der Werf et al. (2009).

The computed versus measured (uniform sediment) results in terms of the net periodaveraged transport are depicted in Figure 13. As seen, a reasonable match is achieved with both data sets (especially for the cases yielding the largest transport rates), hence confirming similar accuracy of the present sediment transport formulation to predict the wave-induced transport for acceleration-skewed flows, as was found for the velocity-skewed flows considered previously. The results in Figure 13 are especially accurate for those cases having the largest transport rates. As was also generally found in the experiments (apart from a single exception in the considered data from Watanabe and Sato, 2004), the computed transport in all cases is in the positive direction.

\subsection{Graded sediments}

To investigate and study the effects of sediment gradation for the asymmetric wave cases considered, we will finally re-simulate the experimental setups in van der A et al. (2010) with the validated model, but now with graded sediment input made from hypothetical mixtures comprised of their well-sorted sands. For this purpose we use the mixtures listed in Table 3. As seen there, the hypothetical mixtures V1, V2, and V4 closely resemble mixtures X1, X2, and X4 from O'Donoghue and Wright (2004a) (see Table 1) and thus are given very similar properties $\left(d_{50}\right.$ is reduced slightly to $0.27 \mathrm{~mm}$ from $d_{50}=0.28 \mathrm{~mm}$ ). Additionally, we have 
also simulated selected cases using directly mix $\mathrm{S}\left(d_{50}=0.15 \mathrm{~mm}\right)$, as defined in Hassan and Ribberink (2005) (see also Table 2). In the following we will specifically compare the fine sand cases utilizing $d=0.15 \mathrm{~mm}$ from van der A et al. (2010) with additional simulations utilizing the fine-grained mixtures $\mathrm{S}\left(d_{50}=0.15 \mathrm{~mm}\right)$ and V1 $\left(d_{50}=0.19 \mathrm{~mm}\right)$. Similarly, we will compare the medium sand cases utilizing $d=0.27 \mathrm{~mm}$ from van der A et al. (2010) with additional simulations utilizing mixtures V2 and V4 (both having $d_{50}=0.27 \mathrm{~mm}$ ).

The comparison (in terms of period-averaged transport rates versus acceleration skewness $\beta$ ) of the van der $A$ et al. (2010) find sand $(d=0.15 \mathrm{~mm})$ cases and those using the fine grain mixtures S and V1 are presented in Figures 14, 15a, 16a, and 17. Each of these figures group results sharing the same wave period $T$ and similar velocity magnitude, hence effectively isolating the effect of variable acceleration skewness $\beta$ on the sediment transport rate. This maintains the presentation style originally utilized by van der A et al. (2010). It is seen that the simulated uniform sediment grain results with $d=0.15 \mathrm{~mm}$ match those measured quite reasonably, in accordance with Figure 13. All results (experimental, as well as those simulated using uniform grains and mixtures) clearly demonstrate essentially a linear increase in the transport rate with acceleration skewness $\beta$.

Direct comparison of the computed results utilizing the fine sediment mixtures with those based on a single grain size suggests that, for these conditions, the added effects of gradation would result in reduced transport rates (by up to a factor 2). This can be explained as follows: Detailed inspection of the results has shown that the total transport of the sediment mixtures is dominated by suspended sediment transport of the fine sand fractions, which again comprise $60 \%$ by weight for both mixtures S and V1 (see again Table $3)$. Since for these mixtures the finest grain sizes $(d=0.13 \mathrm{~mm}$ and $0.15 \mathrm{~mm})$, are quite similar to the grain size considered in the uniform-grain cases $(d=0.15 \mathrm{~mm})$, the resulting net transport rates are, approximately, equal to the uniform fine sand transport rate multiplied by the corresponding fine grain weight fraction. The presence of the other (medium and coarse) sand fractions, effectively shield the amount of fine grained material going into suspension, relative to the uniform fine sand scenario under the same flow conditions.

Table 3: Mixture descriptions for re-simulation of velocity skewed signals of van der A et al. (2010) using graded sediments. Note that mix $\mathrm{S}$ is the same as in Table 2.

\begin{tabular}{cccc}
\hline Mix & $d_{50}(\mathrm{~mm})$ & grain diameters $(\mathrm{mm})$ & weight fractions \\
\hline S & 0.15 & {$[0.13,0.34,0.97]$} & {$[0.60,0.20,0.20]$} \\
V1 & 0.19 & {$[0.15,0.27,0.46]$} & {$[0.60,0.30,0.10]$} \\
V2 & 0.27 & {$[0.15,0.27,0.46]$} & {$[0.20,0.60,0.20]$} \\
V4 & 0.27 & {$[0.15,0.46]$} & {$[0.50,0.50]$} \\
\hline
\end{tabular}

The results for the medium sand cases are similarly depicted in Figures $15 b$ and $16 b$. Interestingly, and in contrast to the fine grain cases just discussed, these results suggest that the added gradation effects are either negligible (in terms of the total sediment transport, e.g. Figure 15b) or can actually result in slightly increased net transport rates (Figure 16b), relative to the uniform medium sand grain scenario. Detailed analysis and inspection of these results has revealed the following qualitative difference: For the results presented in Figure $15 b\left(u_{\max } \approx 1.3 \mathrm{~m} / \mathrm{s}\right)$ the flow is sufficiently strong to induce considerable suspended sediment transport of the median grain size $d=0.27 \mathrm{~mm}$; This likewise accounts e.g. for the 
factor two increase in transport rates seen in Figure $15 b$ relative to Figure 16b. Alternatively, the somewhat weaker flows comprising Figure $16 b\left(u_{\max } \approx 1.1 \mathrm{~m} / \mathrm{s}\right)$ are more dominated by the suspended transport of the fine sand fraction. This observation likewise explains the significantly increased predicted net transport for mixture V4 (50\% fine sand) relative to mixture V2 (20\% fine sand) within Figure $16 b$.

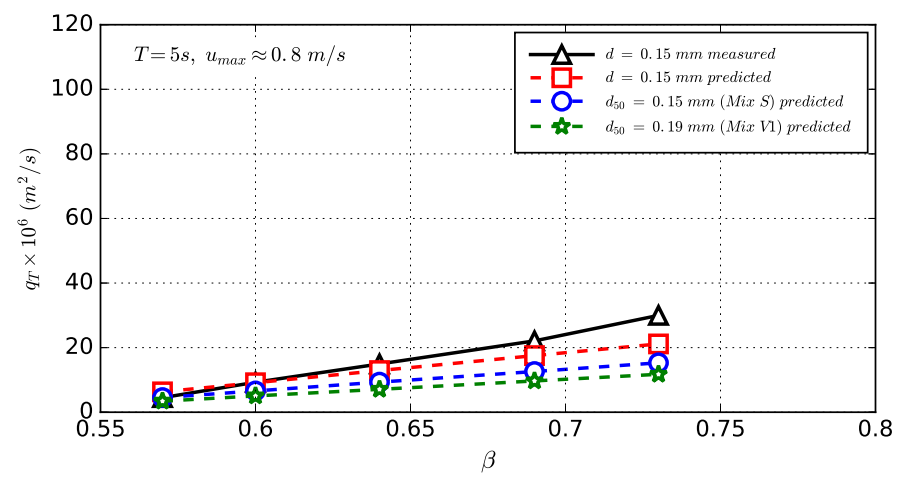

Figure 14: Measured (van der A et al., 2010) and predicted period-averaged net transport rates versus acceleration skewness $\beta$ for $T=5 \mathrm{~s}$ and $d=0.15 \mathrm{~mm}$.

Collectively, the results in this sub-section suggest the potential for gradation effects to increase, decrease, or maintain similar transport rates when compared to uniform grain scenarios having size $d$ similar or equivalent to $d_{50}$ characterizing a sediment mixture. Uniform fine grain sands, in many ways, represent the optimal conditions for suspended sediment transport; The addition of medium and coarse grain fractions in a fine-grain mixture can comparatively reduce transport rates by effectively limiting the amount of fine grained material put into suspension. Alternatively, the present results suggest that medium sediment mixtures (based on their composite $d_{50}$ ) can potentially lead to enhanced transport rates, relative to the uniform grain scenario. This could be expected in situations where the suspended transport of the fine sand fraction in a mixture dominates the transport of the coarser (medium and coarse sand) fractions. Obviously, these findings await direct experimental verification.

\section{Conclusions}

A one-dimensional vertical (1DV) numerical boundary layer and sediment transport model is presented. The model is based on the horizontal component of the Reynoldsaveraged Navier-Stokes (RANS) equations, coupled with two-equation $k$ - $\omega$ turbulence closure (Wilcox, 2006), which in turn drive bed and suspended sediment transport. The developed model significantly extends the uniform grain size model of Fuhrman et al. (2013) to incorporate multiple grain fractions e.g. for simulations involving well-graded sediment mixtures. The model is based on individual bed and suspended load (based on turbulentdiffusion equation) descriptions for each grain fraction, and likewise includes so-called "high concentration effects" of turbulence suppression as well as hindered settling velocities. The model additionally accounts for: 1) increased flow exposure of coarse grain fractions on 

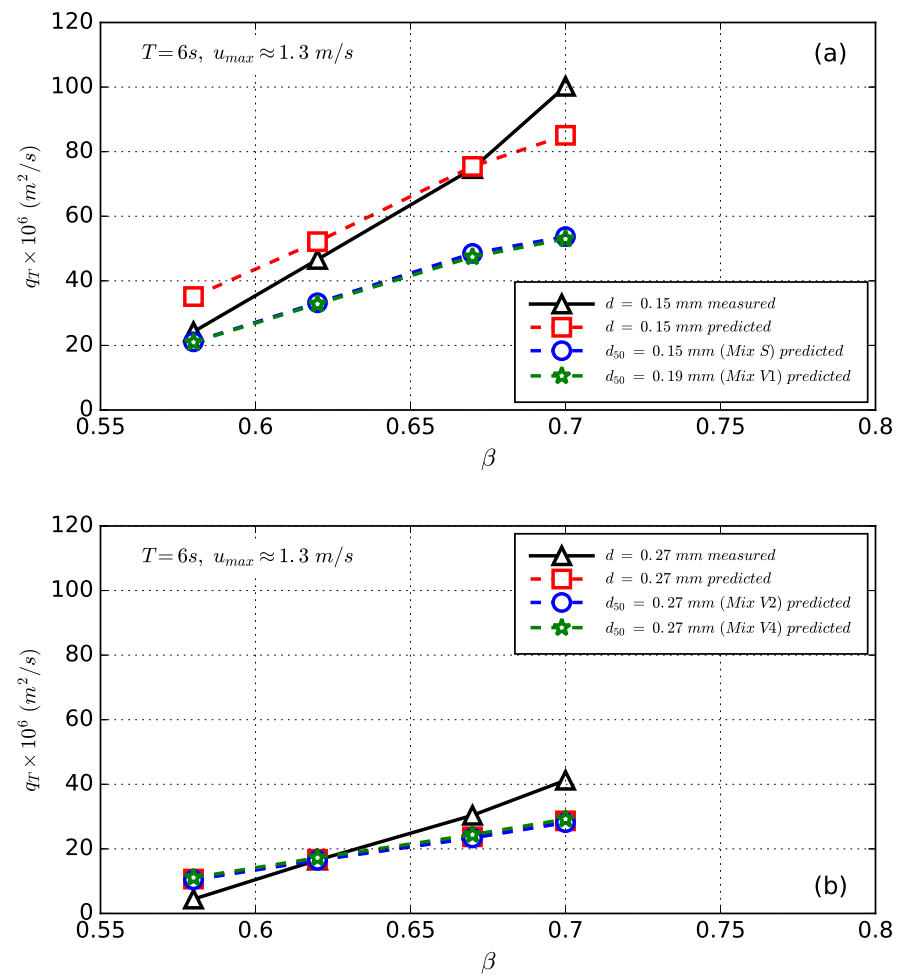

Figure 15: Measured (van der A et al., 2010) and predicted period-averaged net transport rates versus acceleration skewness $\beta$ for $T=6 \mathrm{~s}$ and $(a) d=0.15 \mathrm{~mm}$ and $(b) d=0.27 \mathrm{~mm}$.

well-graded sediment beds, as well as 2) modification of the reference concentrations for applications at a fixed reference level (herein universally based on the median grain size for a given mixture i.e. placed at $b=2 d_{50}$ ). Parametric testing has revealed the importance of these factors, which combine to help alleviate the systematic over- and under-prediction of transported fine and coarse sand fractions, respectively.

As validation of the model net sediment transport rate predictions have been compared with those from oscillating tunnel measurements of O'Donoghue and Wright (2004b) and Hassan and Ribberink (2005), who considered the transport of both well-sorted and graded sediments beneath velocity skewed wave signals. The model demonstrates acceptable accuracy (predictions generally within a factor of two) for the predicted transport of both uniform, as well as graded, sediments. This is based on comparison with both total net transport measurements (O'Donoghue and Wright, 2004b; Hassan and Ribberink, 2005), as well as the net transports of individual grain fractions (Hassan and Ribberink, 2005). Detailed comparison with the graded sediment experiments of Hassan and Ribberink (2005) has confirmed a linear growth of the total net sediment transport versus the third moment of the free stream velocity for mixtures free of fine sand. The model likewise predicts a deviation from this linear trend for mixtures involving fine sand, consistent with experimental observations. The reason for this deviation has been confirmed as due to unsteady phase lag effects, which can reverse the dominant transport direction (to negative) of the fine sand fraction. 

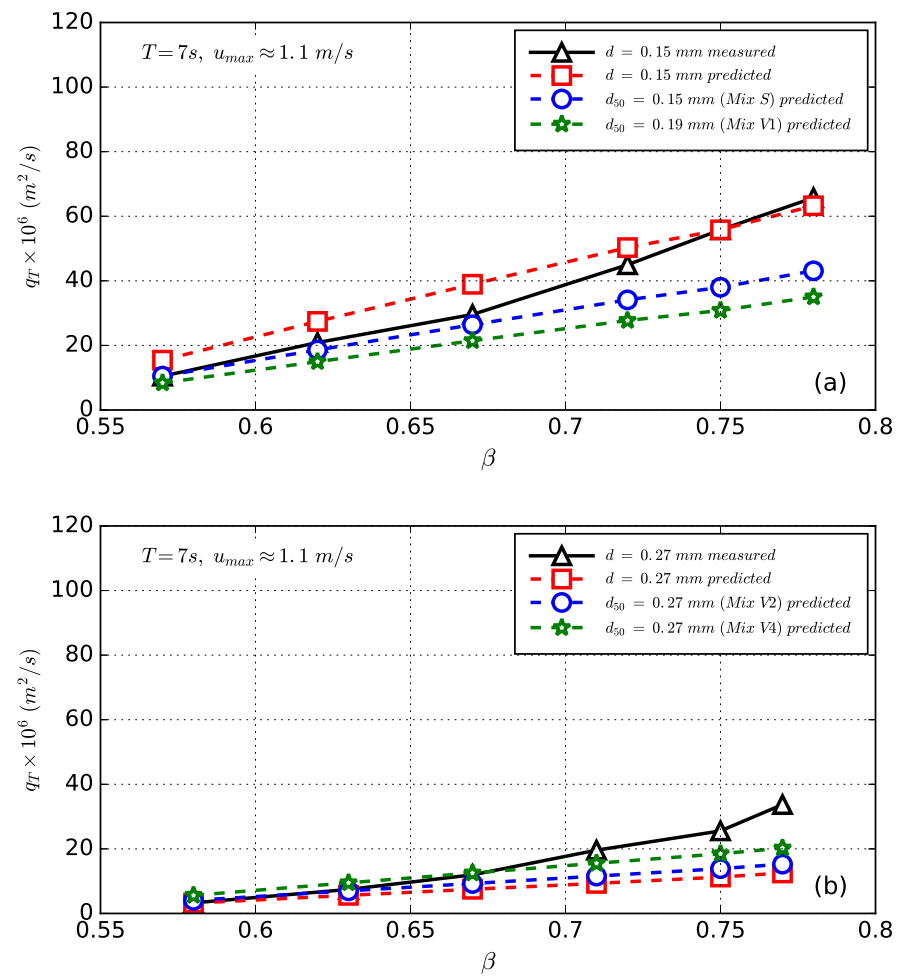

Figure 16: Measured (van der A et al., 2010) and predicted period-averaged net transport rates versus acceleration skewness $\beta$ for $T=7 \mathrm{~s}$ and $(a) d=0.15 \mathrm{~mm}$ and $(b) d=0.27 \mathrm{~mm}$.

Sediment transport beneath asymmetric (acceleration-skewed) wave signals has also been considered. Comparison against the net transport measurements of Watanabe and Sato (2004) and van der A et al. (2010) involving uniform sands have demonstrated similar accuracy as for the previously considered velocity-skewed signals. The validated model has additionally been utilized to study the potential effects of gradation in the transport of sediments beneath such acceleration-skewed flows. Results suggest that gradation effects can both decrease, as well as increase, the total net transport rates relative to uniform sediment beds, depending somewhat on the situation. Model results suggest that for fine sand mixtures (as characterized by their $d_{50}$ ) dominated by suspended sediment transport of the fine sand fraction, gradation effects will tend to reduce net transport rates. This is because the coarser grain fractions tend to limit (shield) the amount of fine material that can be put into suspension. Conversely, for medium sand mixtures (again, as characterized by their $d_{50}$ ) model results suggest that gradation effects can increase the net transport of sediments relative to a uniform medium sand bed, particularly in cases where the suspended transport of the fine sand fraction dominates those of the larger (medium and coarse sand) fractions.

The model developed in the present work is a multi-grain extension to the "MatRANS" model originally developed by Fuhrman et al. (2013). Upon publication, the code (developed in the Matlab environment, complete with numerous example set ups) will be made freely available to others, upon request to the corresponding author. 


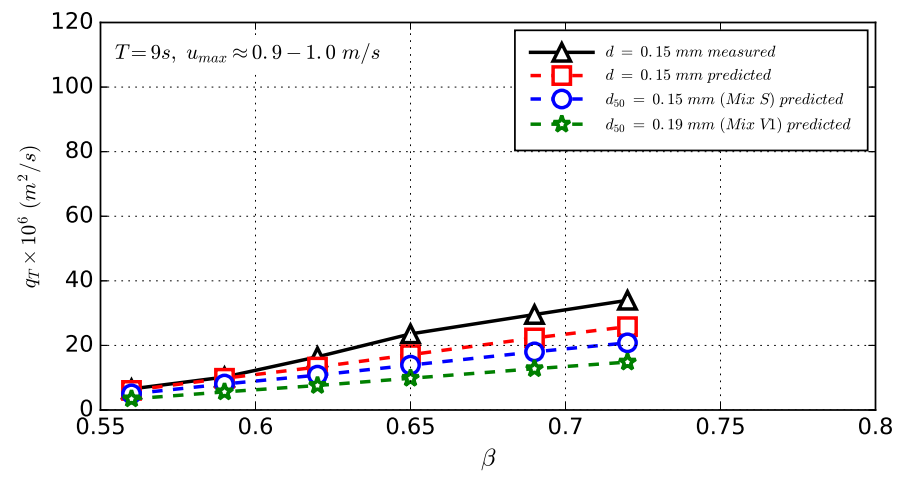

Figure 17: Measured (van der A et al., 2010) and predicted period-averaged net transport rates versus acceleration skewness $\beta$ for $T=9 \mathrm{~s}$ and $d=0.15 \mathrm{~mm}$.

\section{References}

Abreu, T., Silva, P. A., Sancho, F., Temperville, A., 2010. Analytical approximate wave form for asymmetric waves. Coast. Eng. 57, 656-667.

Amoudry, L., Hsu, T.-J., Liu, P. L.-F., 2008. Two-phase model for sand transport in sheet flow regime. J. Geophys. Res. 113, C03011.

Baykal, C., Sumer, B. M., Fuhrman, D. R., Jacobsen, N. G., Fredsøe, J., 2015. Numerical investigation of flow and scour around a vertical circular cylinder. Phil. Trans. Roy. Soc. A 373, article no. 20140104.

Blondeaux, P., Vittori, G., Bruschi, A., Lalli, F., Pesarino, V., 2012. Steady streaming and sediment transport at the bottom of sea waves. J. Fluid Mech. 697, 115-149.

Davies, A. G., Li, Z., 1997. Modelling sediment transport beneath regular symmetrical and asymmetrical waves above a plane bed. Cont. Shelf Res. 17, 555-582.

Engelund, F., Fredsøe, J., 1976. A sediment transport model for straight alluvial channels. Nordic Hydrology 7, 293-306.

Fredsøe, J., Deigaard, R., 1992. Mechanics of Coastal Sediment Transport. World Scientific, Singapore.

Fuhrman, D. R., Baykal, C., Sumer, B. M., Jacobsen, N. G., Fredsøe, J., 2014. Numerical simulation of wave-induced scour and backfilling processes beneath submarine pipelines(submitted).

Fuhrman, D. R., Dixen, M., Jacobsen, N. G., 2010. Physically-consistent wall boundary conditions for the $k-\omega$ turbulence model. J. Hydraul. Res. 48, 793-800.

Fuhrman, D. R., Schløer, S., Sterner, J., 2013. RANS-based simulation of turbulent wave boundary layer and sheet-flow sediment transport processes. Coast. Eng. 73, 151-166. 
Guizien, K., Dohmen-Janssen, M., Vittori, G., 2003. 1DV bottom boundary layer modeling under combined wave and current: Turbulent separation and phase lag effects. J. Geophys. Res. 108, C1, 3016.

Hamm, L., Katapodi, I., Dohmen-Janssen, M., Ribberink, J. S., Samothrakis, P., Cloin, B., Savioli, J. C., Chatelus, Y., Bosboom, J., Nicholson, J., Hein, R., 1998. Grain size, gradation and density effects on sediment transport processes in oscillatory flow conditions. Data report, Part I. WL Delft Hydraulics, The Netherlands.

Hassan, W. N., Ribberink, J. S., 2005. Transport processes of uniform and mixed sands in oscillatory sheet flow. Coast. Eng. 52, 745-770.

Hassan, W. N. M., Ribberink, J. S., 2010. Modelling of sand transport under wave-generated sheet flows with a RANS diffusion model. Coast. Eng. 57, 19-29.

Holmedal, L. E., Myrhaug, D., 2006. Boundary layer flow and net sediment transport beneath asymmetrical waves. Cont. Shelf Res. 26, 252-268.

Holmedal, L. E., Myrhaug, D., 2009. Wave-induced steady streaming, mass transport and net sediment transport in rough turbulent ocean bottom boundary layers. Cont. Shelf Res. 29, 911-926.

Hsu, T.-J., Liu, P. L.-F., 2004. Toward modeling turbulent suspension of sand in the nearshore. J. Geophys. Res. 109, C06018.

Inui, T., Dibajnia, M., Isobe, M., Watanabe, A., 1995. A transport rate formula for mixedsize sands and its application. In: Proceedings of the 42nd Japanese Annual Conference on Coastal Engineering. pp. 356-360, in Japanese.

Jacobsen, N. G., Fredsøe, J., 2014. Formation and development of a breaker bar under regular waves. Part 2: Sediment transport and morphology. Coast. Eng. 88, 55-68.

Kranenburg, W. M., Ribberink, J. S., Schretlen, J. L. L. M., Uittenbogaard, R. E., 2013. Sand transport beneath waves: The role of progressive wave streaming and other free surface effects. J. Geophys. Res. 118, 122-139.

Li, Z., Davies, A. G., 2001. Turbulence closure modelling of sediment transport beneath large waves. Cont. Shelf Res. 21, 243-262.

Liang, D., Cheng, L., 2005. Numerical model for wave-induced scour below a submarine pipeline. J. Waterw. Port C-ASCE 131, 193-202.

Nielsen, P., Callaghan, D. P., 2003. Shear stress and sediment transport calculations for sheet flow under waves. Coast. Eng. 47, 347-354.

O'Donoghue, T., Wright, S., 2004a. Concentrations in oscillatory sheet flow for well sorted and graded sands. Coast. Eng. 50, 117-138.

O'Donoghue, T., Wright, S., 2004b. Flow tunnel measurements of velocities and sand flux in oscillatory sheet flow for well-sorted and graded sands. Coast. Eng. 51, 1163-1184. 
Richardson, J. F., Zaki, W. N., 1954. Sedimentation and fluidisation, Part 1. Trans. Instn. Chem. Engrs. 32, 35-53.

Ruessink, B. G., van den Berg, T. J. J., van Rijn, L. C., 2009. Modeling sediment transport beneath skewed asymmetric waves above a plane bed. J. Geophys. Res. 114, C11021.

Silva, P. A., Abreau, T., van der A, D. A., Sancho, F., Ruessink, B. G., van der Werf, J., Ribberink, J. S., 2011. Sediment transport in nonlinear skewed oscillatory flows: Transkew experiments. J. Hydraul. Res. 49, 72-80.

Sumer, B. M., Chua, L. H. C., Cheng, N.-S., Fredsøe, J., 2003. Influence of turbulence on bed load sediment transport. J. Hydraul. Eng. ASCE 129, 585-596.

van der A, D. A., O’Donoghue, T., Ribberink, J. S., 2010. Measurements of sheet flow transport in acceleration-skewed oscillatory flow and comparison with practical formulations. Coast. Eng. 57, 331-342.

van der A, D. A., Ribberink, J. S., van der Werf, J. J., O'Donoghue, T., Buijsrogge, R. H., Kranenburg, W. M., 2013. Practical sand transport formula for non-breaking waves and current. Coast. Eng. 76, 26-42.

van der Werf, J. J., Schretlen, J. J. L. M., Ribberink, J. S., O’Donoghue, T., 2009. Database of full-scale laboratory experiments on wave-driven sand transport processes. Coast. Eng. $56,726-732$.

van Rijn, L. C., 2007. Unified view of sediment transport by currents and waves. III: Graded beds. J. Hydraul. Eng. 133, 761-775.

Watanabe, A., Sato, S., 2004. A sheet-flow transport rate formula for asymmetric forwardleaning waves and currents. In: Proceedings of the 29th International Conference on Coastal Engineering. Vol. 2. Lisbon, pp. 1703-1714.

Wilcox, D. C., 2006. Turbulence Modeling for CFD, 3rd Edition. DCW Industries, Inc., La Canada, California.

Wilcox, D. C., 2008. Formulation of the $k-\omega$ turbulence model revisited. AIAA J. 46, 28232838 .

Williams, I. A., Fuhrman, D. R., 2016. Numerical simulation of tsunami-scale wave boundary layers. Coast. Eng. 110, 17-31.

Wu, W., Lin, W., 2014. Nonuniform sediment transport under non-breaking waves and current. Coast. Eng. 90, 1-11.

Zyserman, J. A., Fredsøe, J., 1994a. Bed concentration of suspended sediment and total load transport in asymmetric oscillatory flow. In: Book of Abstracts, Overall Workshop MAST. Gregynog, Wales.

Zyserman, J. A., Fredsøe, J., 1994b. Data analysis of bed concentration of suspended sediment. J. Hydraul. Eng. 120, 1021-1042. 


\section{Appendix A. Derivation and discussion of the reference concentration modifi- cation (22)}

In this appendix a derivation of the modification applied to the reference concentration is provided, for the simple case involving steady uniform flow. In this case, neglecting high-concentration effects for simplicity, the turbulent-diffusion equation (19) simplifies to

$$
c_{i} w_{s 0, i}+\epsilon_{s} \frac{\partial c_{i}}{\partial y}=0
$$

Assuming a linearly growing eddy viscosity of the form

$$
\epsilon_{s}=\kappa U_{f} \beta_{s} y
$$

where $\kappa=0.4$ is the von Karman constant, yields the well-known solution for the suspended sediment concentration distribution

$$
c_{i}=c_{b 0, i}\left(\frac{b_{i}}{y}\right)^{Z_{i}}, \quad Z_{i}=\frac{w_{s 0, i}}{\kappa U_{f} \beta_{s}} .
$$

Here $Z_{i}$ is the Rouse parameter for the $i$ th grain fraction, and $b_{i}$ is taken as the "natural" reference level for a given grain size i.e. $b_{i}=2 d_{i}$, such that $c_{i}\left(y=b_{i}\right)=c_{b 0, i}$.

Consider now the modification required to still (approximately) satisfy that $c_{i}\left(y=b_{i}\right)=$ $c_{b 0, i}$, if the actual reference level is taken at some other position, e.g. fixed at $b=2 d_{50} \neq b_{i}$. Defining the actual reference concentration as $c_{b, i}=c_{i}(y=b)$, (A.3) becomes

$$
c_{b, i}=c_{b 0, i}\left(\frac{b_{i}}{b}\right)^{Z_{i}}
$$

Notice that this expression is precisely of the form (22), and that the parameter $\Gamma$ is clearly related to the respective Rouse parameter $Z_{i}$ at the limit of steady uniform flow (which is obviously a special case of more complicated unsteady flow situations). For simplicity, in the unsteady simulations considered in the present work, we have again utilized fixed $\Gamma=1.25$, though this physical link with the Rouse parameter is emphasized, as it implies that this correction factor is, mostly likely, not a global constant.

The modification described above and leading to (22) is, essentially, nothing more than a means of translating reference concentrations (conceptually) computed individually for multiple grain fractions to a common reference level $b=2 d_{50}$, hence conveniently enabling use of a single computational grid for the simulation of suspended sediment concentrations for all fractions. This modification is reasonable, provided the grain sizes comprising a mixture are reasonably similar in size. It should be cautioned, however, that in extreme circumstances (e.g. widely differing grain sizes coupled with large Shields parameters for all fractions) application of (22) could potentially result in un-physically large concentrations. In such circumstances reducing $\Gamma$, switching this feature off altogether (setting $\Gamma=0$ ), or e.g. further extension to use individual grids for each suspended fraction (i.e. each with their own reference level $b_{i}$ ), would be preferable/necessary. It is emphasized that this issue has seemingly not been overtly problematic in any of the cases considered in the present work (corresponding to full scale waves with mixed sand fractions in the sheet flow 
transport regime): The largest total suspended sediment concentration encountered in any of the sheet-flow simulations considered herein has been $c=0.44$ (case S4 of Hassan and Ribberink, 2005), whereas the total concentration has not exceeded $c=0.35$ in any of the other cases. 